TRANSACTIONS OF THE

AMERICAN MATHEMATICAL SOCIETY

Volume 357, Number 2, Pages 757-792

S 0002-9947(04)03498-1

Article electronically published on May 10, 2004

\title{
THE DIRICHLET PROBLEM FOR HARMONIC MAPS FROM RIEMANNIAN POLYHEDRA TO SPACES OF UPPER BOUNDED CURVATURE
}

\author{
BENT FUGLEDE
}

In memory of Professor Heinz Bauer

\begin{abstract}
This is a continuation of the Cambridge Tract "Harmonic maps between Riemannian polyhedra", by J. Eells and the present author. The variational solution to the Dirichlet problem for harmonic maps with countinuous boundary data is shown to be continuous up to the boundary, and thereby uniquely determined. The domain space is a compact admissible Riemannian polyhedron with boundary, while the target can be, for example, a simply connected complete geodesic space of nonpositive Alexandrov curvature; alternatively, the target may have upper bounded curvature provided that the maps have a suitably small range. Essentially in the former setting it is further shown that a harmonic map pulls convex functions in the target back to subharmonic functions in the domain.
\end{abstract}

\section{INTRODUCTION AND PRELIMINARIES}

While interior regularity (Hölder continuity) of locally energy minimizing maps from an admissible Riemannian polyhedron $(X, g)$ was the subject of the article [F3] - an addendum to the recent monograph [EF] by J. Eells and the author - the present paper is concerned with the Dirichlet problem for harmonic (i.e., continuous locally energy minimizing) maps of $(X, g)$ into a complete geodesic space $\left(Y, d_{Y}\right)$ of nonpositive curvature (Theorem 1) or of upper bounded curvature (Theorem 3), assuming that $X$ is compact and has nonvoid boundary $b X$. As an application in the former setting, two pull-back properties of harmonic maps are included (Theorem 2). I am indebted to Professor Eells for posing the problems treated in this article to me back in 1996 .

Upper bounds $(\geq 0)$ for the curvature of $Y$ are understood in the sense of A.D. Alexandrov [A1, A2. Existence and uniqueness of the variational solution $\phi: X \rightarrow Y$ was obtained in [EF], [F4], and Hölder continuity of $\phi$ off the boundary in $[\mathrm{EF}$, [F3]. A few references to related known results for the particular case of maps from a Riemannian manifold will be given below in Section 2 .

In Part (a) of Theorems 1 and 3 we show that the variational solution $\phi$ is continuous up to the boundary $b X$ provided that the prescribed boundary map is continuous and, in Theorem 3 (a), that $Y$ is locally compact. In Part (b) of either

Received by the editors February 18, 2003 and, in revised form, September 2, 2003.

2000 Mathematics Subject Classification. Primary 58E20, 49N60; Secondary 58A35.

Key words and phrases. Harmonic map, Dirichlet problem, Riemannian manifold, Riemannian polyhedron, geodesic space, Alexandrov curvature.

(C) 2004 American Mathematical Society 
theorem we establish uniqueness of a harmonic map with prescribed continuous restriction to the boundary. This is done by proving the same maximum principle for the distance function $d_{Y}\left(\phi_{0}, \phi_{1}\right)$ between two harmonic maps $\phi_{0}, \phi_{1}$ which was obtained by Jäger and Kaul [JK] in the case where $X$ and $Y$ are Riemannian manifolds. Also in our setting, $d_{Y}\left(\phi_{0}, \phi_{1}\right)$ is therefore subharmonic in case $Y$ has nonpositive curvature (Theorem $1(\mathrm{~b})$ ), as shown by considering the map $\phi_{0} \times \phi_{1}$ : $X \rightarrow Y \times Y$. In the case of upper bounded curvature (Theorem $3(\mathrm{~b})), d_{Y}\left(\phi_{0}, \phi_{1}\right)$ is expressed in terms of a subsolution to the very same elliptic operator of divergence type as devised in [JK]. Summing up, the Dirichlet problem is uniquely solvable and well posed.

Similar results are obtained for the Dirichlet problem for maps into a smooth Riemannian manifold without boundary (Theorems 4 and 5). The study of maps into a manifold is not quite a particular case of that of a geodesic space target because our concept of energy of maps into a geodesic space, extending that of Korevaar and Schoen [KS] for maps from a (smooth) Riemannian manifold, requires that the Riemannian metric $g$ on the domain polyhedron $X$ be simplexwise smooth, while bounded measurable components of $g$ suffice for a good concept of energy of maps into a Riemannian manifold. When $g$ is simplexwise smooth, the two concepts of energy are identical (up to a dimensional constant factor) provided either that $\phi(X)$ is bounded $\mathrm{EF}$ Theorem 9.2] or that the target manifold is complete and simply connected with nonpositive sectional curvature (Proposition 2 below).

Recall from [EF, Chapter 4] that a polyhedron $X$ is termed admissible if it is dimensionally homogeneous, say of dimension $m$, and if (in case $m \geq 2$ ) any two $m$-simplexes of $X$ with a common face $\sigma(\operatorname{dim} \sigma=0,1, \ldots, m-2)$ can be joined by a chain of $m$-simplexes containing $\sigma$, any two consecutive ones of which have a common $(m-1)$-face containing $\sigma$.

We denote throughout by $X$ an $m$-dimensional admissible polyhedron, connected and locally finite, and endowed with a nondegenerate Riemannian metric $g$ whose restriction to each open $m$-simplex of $X$ is at least bounded and measurable. The associated volume measure on $X$ is denoted by $\mu_{g}=\mu$, the intrinsic (Riemannian) distance on $X$ by $d_{X}^{g}=d_{X}$, and the closed ball with center $x \in X$ and radius $r$ by $B_{X}(x, r)$.

Based on the work of Korevaar and Schoen [KS] a concept of energy of a map $\phi$ of $(X, g)$ into a metric space $\left(Y, d_{Y}\right)$ is developed in [EF, Chapter 9], assuming that $g$ is simplexwise smooth, i.e., $g$ shall be smooth in every open $m$-simplex $s$ of $X$, and $g_{\mid s}$ shall extend smoothly to a (nondegenerate) Riemannian metric on the affine $m$-space containing $s$ EF, Remark 4.1]. The map $\phi$ is supposed first of all to be $\mu$-measurable with separable essential range, and to be of class $L_{\text {loc }}^{2}(X, Y)$ in the sense that the distance function $d_{Y}(\phi(\cdot), y)$ is of class $L_{\text {loc }}^{2}(X, \mu)$ for some and hence for any point $y \in Y$. The approximate energy density $e_{\epsilon}(\phi) \in L_{\mathrm{loc}}^{1}(X, \mu)$ is then defined for $\epsilon>0$ at every point $x \in X$ by

$$
e_{\epsilon}(\phi)(x)=\int_{B_{X}(x, \epsilon)} \frac{d_{Y}^{2}\left(\phi(x), \phi\left(x^{\prime}\right)\right)}{\epsilon^{m+2}} d \mu\left(x^{\prime}\right) .
$$

Definition 1. The energy of $\phi:(X, g) \rightarrow\left(Y, d_{Y}\right)$ is

$$
E(\phi)=\sup _{f \in C_{c}(X,[0,1])}\left(\limsup _{\epsilon \rightarrow 0} \int_{X} f e_{\epsilon}(\phi) d \mu\right) \quad(\leq \infty),
$$


where $C_{c}$ stands for continuous functions of compact support. $W_{\text {loc }}^{1,2}(X, Y)$ denotes the space of all maps $X \rightarrow Y$ for which $E\left(\phi_{\mid U}\right)<\infty$ for every relatively compact connected open set $U \subset X$ (equivalently, the above limsup is finite for every $f$ ).

We shall sometimes use the fact that the supremum over $f$ in (1.2) is also the limit of the increasing net in question with the upper directed index set $C_{c}(X,[0,1])$ (ordered pointwise). If $X$ is compact, then (1.2) reduces to

$$
E(\phi)=\limsup _{\epsilon \rightarrow 0} \int_{X} e_{\epsilon}(\phi) d \mu .
$$

It is shown that, if $\phi \in W_{\mathrm{loc}}^{1,2}(X, Y)$ (and only then), there exists a non-negative function $e(\phi) \in L_{\mathrm{loc}}^{1}(X, \mu)$, called the energy density of $\phi$, such that $e_{\epsilon}(\phi) \rightarrow e(\phi)$ as $\epsilon \rightarrow 0$, in the sense of weak convergence as measures

$$
\lim _{\epsilon \rightarrow 0} \int_{X} f e_{\epsilon}(\phi) d \mu=\int_{X} f e(\phi) d \mu
$$

for every $f \in C_{c}(X)$. In the affirmative case it follows from (1.2), (1.3) that

$$
E(\phi)=\int_{X} e(\phi) d \mu
$$

For the above assertions, see Steps 2, 3, and 4 of the proof of [EF, Theorem 9.1]. These steps are independent of the general requirement in $[\mathrm{EF}]$ that also the target of maps $X \rightarrow Y$ shall be locally compact. However, the proof of the second part of Step 1, leading to quasicontinuity of $\phi$, required compactness of closed balls in $Y$. Actually, this extra requirement can be replaced by $\left(Y, d_{Y}\right)$ being complete: Every map $\phi \in W_{\text {loc }}^{1,2}(X, Y)$, with $\left(Y, d_{Y}\right)$ a complete metric space, has a quasicontinuous version [F4].

A function $u: X \rightarrow \mathbb{R}$ is of class $W_{\text {loc }}^{1,2}(X, \mathbb{R})$ in the sense of Definition 1 (with $Y=\mathbb{R}$ ) if and only if $u \in W_{\text {loc }}^{1,2}(X)$ as defined in [EF, p. 63] (cf. [F3 note 2, p. 377] for the uniqueness of $\nabla u$ ). And if that is the case, the energy density of $u$ equals

$$
e(u)=c_{m}|\nabla u|^{2}=c_{m} g^{i j} \partial_{i} u \partial_{j} u \quad \text { a.e. in } X,
$$

with the usual summation convention. Here $c_{m}=\omega_{m} /(m+2), \omega_{m}$ being the volume of the unit ball in $\mathbb{R}^{m}$. See [EF] Corollary 9.2], which is based on [KS, Theorem 1.6.2] (where $X$ is a Riemannian domain in a Riemannian manifold), and is also a particular case of [EF, Theorem 9.2].

By polarization, (1.3) and (1.4) lead, for any two functions $u, v \in W_{\text {loc }}^{1,2}(X)$, to

$$
\int_{B_{X}(x, \epsilon)} \frac{\left(u(x)-u\left(x^{\prime}\right)\right)\left(v(x)-v\left(x^{\prime}\right)\right)}{\epsilon^{m+2}} d \mu\left(x^{\prime}\right) \rightarrow c_{m}\langle\nabla u(x), \nabla v(x)\rangle
$$

as $\epsilon \rightarrow 0$, in the sense of weak convergence as measures.

For any map $\phi \in W_{\mathrm{loc}}^{1,2}(X, Y)$ and any connected open set $U \subset X$ it is easily shown that $e\left(\phi_{\mid U}\right)=e(\phi) \mu$-a.e. in $U$, and so $E\left(\phi_{\mid U}\right)=\int_{U} e(\phi) d \mu$. It follows that, if two maps $\phi, \psi \in W_{\mathrm{loc}}^{1,2}(X, Y)$ agree $\mu$-a.e. off a closed set $F \subset X$, then $e(\phi)=e(\psi)$ $\mu$-a.e. in $X \backslash F$, and hence (if $E(\phi)<\infty$ )

$$
E(\psi)-E(\phi)=\int_{F}(e(\psi)-e(\phi)) d \mu .
$$

A map $\phi \in W_{\text {loc }}^{1,2}(X, Y)$ is said to be locally E-minimizing, or to be a local Eminimizer, if $X$ can be covered by relatively compact subdomains $U$ for each of 
which $E\left(\phi_{\mid U}\right) \leq E\left(\psi_{\mid U}\right)$ holds for every map $\psi \in W_{\text {loc }}^{1,2}(X, Y)$ such that $\psi=\phi \mu$ a.e. in $X \backslash U[\mathrm{EF}$, Definition 10.1]. It would clearly amount to the same to consider instead maps $\psi$ such that $\psi=\phi \mu$-a.e. off some closed set $F \subset U$ ( $F$ depending on $\psi$ ). If $E(\phi)<\infty$ (e.g. if $X$ is compact), then the inequality $E\left(\phi_{\mid U}\right) \leq E\left(\psi_{\mid U}\right)$ may therefore be replaced equivalently by $E(\phi) \leq E(\psi)$, according to (1.6).

In the setting of any one of the five theorems below, a continuous local E-minimizer $\phi: X \rightarrow Y$ is called a harmonic map; see [EF, Chapter 12].

\section{Formulation of Results}

We proceed to formulate and comment on the results of the present article. Proofs are mostly given in subsequent sections. The admissible Riemannian polyhedron $(X, g)$ of dimension $m$ is supposed (in the absence of another indication) to be compact with nonempty boundary $b X$, the union of all $(m-1)$-simplexes of $X$ contained in only one $m$-simplex. It follows that $X \backslash b X$ is noncompact.

Maps into a geodesic space. In this subsection we suppose that $g$ is simplexwise smooth (as explained in the paragraph containing (1.1)). To begin with, $\left(Y, d_{Y}\right)$ is just a complete metric space. Then so is $L^{2}(X, Y)$, endowed with the metric $D$ given by $D^{2}(\phi, \psi):=\int_{X} d_{Y}^{2}(\phi, \psi) d \mu$ for $\phi, \psi \in L^{2}(X, Y)$; cf. [KS, $\left.\S 1.1\right]$.

We denote by $W^{1,2}(X, Y)$ the class of all maps $\phi: X \rightarrow Y$ of finite energy in the sense of Definition 1:

$$
E(\phi)=\int_{X} e(\phi) d \mu=\lim _{\epsilon \rightarrow 0} \int_{X} e_{\epsilon}(\phi) d \mu .
$$

For each $(m-1)$-simplex $\sigma$ of the boundary $b X$, the $\operatorname{trace} \operatorname{tr}_{\sigma} \phi$ on $\sigma$ of a map $\phi \in W^{1,2}(X, Y)$ is well defined as the trace on $\sigma$ of the restriction $\phi_{\mid s}$ of $\phi$ to the unique open $m$-simplex $s$ of $X$ having $\sigma$ as a face; cf. [KS, §1.12]. Note that $\operatorname{tr}_{\sigma} \phi$ is of class $L^{2}(\sigma, Y)$ on $\sigma$ relative to $(m-1)$-dimensional Hausdorff measure $\mathcal{H}_{m-1}$ [KS p. 606]. When collected, the traces $\operatorname{tr}_{\sigma} \phi$ on the various $(m-1)$-simplexes $\sigma$ of $b X$ define the trace $\operatorname{tr}_{b X} \phi$ of $\phi$ on $b X$ as a map of class $L^{2}(b X, Y)$, a complete metric space with metric analogous to $D$ above.

In terms of a quasicontinuous version $\phi$ (cf. [F4, Theorem 1]), the restriction of $\phi$ to $\sigma$ equals $\operatorname{tr}_{\sigma} \phi \mathcal{H}_{m-1}$-a.e. (In fact, the projection on $\sigma$ of a set $\omega \subset s$ along a flow in $s$ transversal to $\sigma$ has capacity $\leq$ a constant times the capacity of $\omega$, and any set of capacity 0 has $\mathcal{H}_{m-1}$-measure 0 ; cf. e.g. [La, Theorems $\left.2.9,3.13\right]$.)

The following lemma, proven in Section 3, is known when $(X, g)$ is a Lipschitz Riemannian domain (in a Riemannian manifold); see [KS, Theorem 1.12.2] for (a) and (b) in that setting.

Lemma 1. (a) If a sequence $\left(\phi_{n}\right) \subset W^{1,2}(X, Y)$ has uniformly bounded energies, and if $\left(\phi_{n}\right)$ converges in $L^{2}(X, Y)$ to a map $\phi$ (necessarily of class $W^{1,2}(X, Y)$ ) then $\operatorname{tr}_{b X} \phi_{n} \rightarrow \operatorname{tr}_{b X} \phi$ in $L^{2}(b X, Y)$.

(b) For any two maps $\phi, \psi \in W^{1,2}(X, Y)$ and any compact set $\Gamma \subset b X, \operatorname{tr}_{\Gamma} \phi=$ $\operatorname{tr}_{\Gamma} \psi$ holds if an only if the distance function $d_{Y}(\phi, \psi): X \rightarrow \mathbb{R}$ (necessarily of class $\left.W^{1,2}(X)\right)$ has trace 0 on $\Gamma$, or equivalently, $d_{Y}(\phi, \psi) \in W_{0}^{1,2}(X \backslash \Gamma)$.

(c) There is a constant $C$ such that the Poincaré-style inequality

$$
\int_{X} u^{2} d \mu \leq C \int_{X}|\nabla u|^{2} d \mu
$$

holds for any function $u \in \operatorname{Lip}_{c}(X \backslash b X)$, hence for any $u \in W_{0}^{1,2}(X \backslash b X)$. 
It follows from (c) that $1 \notin W_{0}^{1,2}(X \backslash b X)$. For a given map $\psi \in W^{1,2}(X, Y)$ consider the subclass

$$
\begin{aligned}
W_{\psi}^{1,2}(X, Y) & =\left\{\phi \in W^{1,2}(X, Y): \operatorname{tr}_{b X} \phi=\operatorname{tr}_{b X} \psi \mathcal{H}_{m-1} \text {-a.e. }\right\} \\
& =\left\{\phi \in W^{1,2}(X, Y): d_{Y}(\phi, \psi) \in W_{0}^{1,2}(X \backslash b X)\right\},
\end{aligned}
$$

the latter equation by Lemma 1 (b) (applied to $\Gamma=b X$ ), which also shows that $d_{Y}\left(\phi_{0}, \phi_{1}\right) \in W_{0}^{1,2}(X \backslash b X)$ for any two maps $\phi_{0}, \phi_{1} \in W_{\psi}^{1,2}(X, Y)$.

For the case of a nonpositively curved target our main result is

Theorem 1. Let $\left(Y, d_{Y}\right)$ be a simply connected complete geodesic space of nonpositive curvature.

(a) For any map $\psi \in W^{1,2}(X, Y)$ the unique map $\phi$ of least energy in $W_{\psi}^{1,2}(X, Y)$ is Hölder continuous in $X \backslash b X$, and continuous at any point of $b X$ at which $\operatorname{tr}_{b X} \psi$ is continuous.

(b) For any two continuous maps $\phi_{0}, \phi_{1}: X \rightarrow Y$ which are harmonic in $X \backslash b X$ the distance function $d_{Y}\left(\phi_{0}, \phi_{1}\right) \in W^{1,2}(X)$ is subharmonic in $X \backslash b X$, and hence attains its maximum on $b X$ :

$$
\max _{X} d_{Y}\left(\phi_{0}, \phi_{1}\right)=\max _{b X} d_{Y}\left(\phi_{0}, \phi_{1}\right)
$$

In particular, if $\phi_{0}=\phi_{1}$ on $b X$, then $\phi_{0}=\phi_{1}$ on $X$.

According to the proof of [EF, Theorem 11.3], $W_{\psi}^{1,2}(X, Y)$ has indeed a unique element $\phi$ of least energy, called the variational solution to the Dirichlet problem on $X$ with boundary map $\psi\left(\operatorname{or} \operatorname{tr}_{b X} \psi\right.$ ). This was proved as in [KS, Theorem 2.2], where $(X, g)$ is a Lipschitz Riemannian domain (in a Riemannian manifold).

It is understood in (a) that $\phi$ has been redefined (uniquely) on a $\mu$-nullset so as to become (Hölder) continuous in $X \backslash b X$; this is possible by [EF, Theorem 10.1], F3. Theorem 1], because $\phi_{\mid X \backslash b X}$ is, in particular, locally $E$-minimizing. See also Jost Jo2 for the underlying technique. It is further understood that $\operatorname{tr}_{b X} \phi=\operatorname{tr}_{b X} \psi$ has been redefined $\mathcal{H}_{m-1}$-a.e. on $b X$ so as to become continuous at the given point $x_{0} \in b X$. The latter claim in (a) is then that the version of $\phi: X \rightarrow Y$ thus obtained (with $\phi_{\mid b X}:=\operatorname{tr}_{b X} \phi$ ) is continuous at $x_{0}[$

From (b) it follows that the variational solution $\phi$ is the only map of class $W_{\psi}^{1,2}(X, Y)$ which is harmonic in $X \backslash b X$. (Use the latter expression (2.1) for $W_{\psi}^{1,2}(X, Y)$ and apply the maximum principle from [EF, Proposition 5.3 (i)] to the subharmonic function $d_{Y}\left(\phi_{0}, \phi_{1}\right)$ on $X \backslash b X$.)

If $\operatorname{tr}_{b X} \psi$ is continuous it follows similarly from (a) and (b), in view of the former expression (2.1) and [EF Proposition 5.3 (ii)], that the variational solution $\phi$ is also the only solution to the classical-style Dirichlet problem of finding a continuous map $\phi$ of class $W_{\psi}^{1,2}(X, Y)$ such that $\phi$ is harmonic in $X \backslash b X$. Thus the classical-style

\footnotetext{
${ }^{1}$ In the proofs of $[\mathrm{EF}$, Theorems 10.1, 10.2] and [F3, Theorem 2], the assertion at the beginning that $X$ may be assumed compact is incorrect and should simply be omitted, having only trivial consequences such as replacing $W^{1,2}(X)$ by $W_{\text {loc }}^{1,2}(X)$ and $\operatorname{Lip}(X)$ by $\operatorname{Lip}_{c}(X)$. The same applies to the proofs of the corresponding results for maps into manifolds $[\mathrm{EF}$ Propositions 12.1, 12.2] and [F3, Theorem 4]. Furthermore, in the beginning of the large display [F3, p. 395], one should accordingly delete the string " $\frac{1}{2} E\left(\phi_{\lambda}\right)-\frac{1}{2} E(\phi)=\frac{1}{2} \int_{F}\left(e\left(\phi_{\lambda}\right)-e(\phi)\right) d \mu=$ " (not used anyway) because $E(\phi)$ may now be infinite. Similarly, on [EF, p. 194], $E\left(\phi_{\lambda}\right)-E(\phi)$ should be replaced by $E\left(\left(\phi_{\lambda}\right)_{\mid U}\right)-E\left(\phi_{\mid U}\right)$.
} 
Dirichlet problem is uniquely solvable, and well posed (in the uniform topology on continuous maps) in view of (2.2).

The question of Hölder continuity of the variational solution $\phi$ in (a) up to the boundary in case $\operatorname{tr}_{b X} \psi$ is Hölder continuous remains open in the present setting of a polyhedral domain $X$. For the case that $(X, g)$ is a Riemannian manifold with smooth boundary, $\phi$ is Hölder continuous up to the boundary with exponent $\alpha$ provided that $\psi$ is Hölder continuous with that exponent $(0<\alpha<1)$ [Se1].

The proof of Theorem 1 is given in Section 4. Part (a) is reduced there to the (nontrivial) case $Y=\mathbb{R}$ of harmonic functions. The key to (b) is to pass to the product map $\phi_{0} \times \phi_{1}: X \rightarrow Y \times Y$ and profit from the isometric involution $\left(y_{0}, y_{1}\right) \mapsto\left(y_{1}, y_{0}\right)$ on $Y \times Y$, thereby greatly simplifying triangle comparison, or rather quadrilateral comparison $[\mathrm{KS}$ \ $\$ 2.1]$ - a particular case of the characterization of nonpositive curvature by comparison of closed curves, due to Reshetnyak [R]. The proof in (b) that $d_{Y}\left(\phi_{0}, \phi_{1}\right)$ is subharmonic (when the maps $\phi_{0}$ and $\phi_{1}$ are harmonic) is given in the slightly more general situation in which $\phi_{0}$ and $\phi_{1}$ are harmonic in an open subset of $X$, or equally well in the entire space $X$, which is then typically noncompact and may have empty boundary.

Remark 1. By comparison with the classical Dirichlet problem for harmonic functions it is natural to replace $X \backslash b X$ in Theorem 1 by a domain $\Omega$ with compact closure and nonvoid boundary in an ambient admissible Riemannian polyhedron $X$. It can be shown that Theorem 1 remains valid in that setting (at least for bounded maps), assuming just that the point of continuity in question in (a) is a regular boundary point in the sense of potential theory, and using the latter definition (2.1) of $W_{\psi}^{1,2}(X, Y)$; similarly as to Theorems 3 , 4, and 5. See [F5].

Our next theme is certain pull-back properties of harmonic maps. The domain polyhedron $X$ is no longer required to be compact and with nonempty boundary (except in Corollary 1).

Theorem 2. Let $\left(Y, d_{Y}\right)$ be a simply connected complete geodesic space of nonpositive curvature, and let $\phi: X \rightarrow Y$ be a harmonic map.

(a) For any closed convex set $C \subset Y$ the shortest distance $d_{Y}(\phi(x), C)$ is subharmonic as a function of $x \in X$.

(b) For any continuous convex function $f$ on an open set $V \subset Y, f \circ \phi$ is subharmonic in $\phi^{-1}(V)$ (supposed nonempty).

Recall that the shortest distance $d_{Y}(y, C)$ between a point $y \in Y$ and the set $C$ from (a) is well defined by

$$
d_{Y}(y, C)=\min _{z \in C} d_{Y}(y, z)
$$

and $d_{Y}(y, C)$ is convex and contractive as a function of $y \in Y$, the former because $\left(Y, d_{Y}\right)$ is globally squared-convex (cf. e.g. [EF, p. 26]), and the latter by [KS, Proposition 2.5.4]. Part (a) is therefore a particular case of Part (b), but needed in our proof of (b). The proof of Theorem 2 is given in Section 5 .

As an immediate consequence of (a) we have a kind of maximum principle for harmonic maps $X \rightarrow Y$ when $X$ is compact and has nonempty boundary $b X$ :

Corollary 1. Let $\left(Y, d_{Y}\right)$ be as in Theorems 1 and 2. For any closed convex set $C \subset Y, \phi(b X) \subset C$ implies $\phi(X) \subset C$. 
Indeed, this means that $d_{Y}(\phi, C)=0$ on $b X$ implies $d_{Y}(\phi, C)=0$ in all of $X$. From Theorem 2 (b) together with Theorem 1 (b) we obtain the following:

Corollary 2. Let $\left(Y, d_{Y}\right)$ be as in Theorems 1 and 2.

(a) A map $\phi \in W_{\text {loc }}^{1,2}(X, Y)$ which is harmonic off some closed polar set $F \subset X$ can be redefined on $F$ so as to become harmonic in all of $X$.

(b) The limit $\phi$ of a locally uniformly convergent, locally E-bounded sequence of harmonic maps $\phi_{n}: X \rightarrow Y$ is harmonic.

Ad (a). For maps between Riemannian manifolds (no curvature requirement) this removable singularity result is due to Eells and Polking [EP]. To prove it in the present setting, let $U$ denote the (open) star of a vertex of $X$ in a triangulation sufficiently fine that $U$ is the interior of its closure; cf. [EF Remark 4.2]. Then $\partial U=b U$, and $\bar{U}$ is compact. Let $\psi$ be the variational solution to the Dirichlet problem in $\bar{U}$ with boundary map $\operatorname{tr}_{b U} \psi=\operatorname{tr}_{b U} \phi$; cf. Theorem 1 (a). Since the stars $U$ cover $X$, it suffices to prove that $\psi=\phi$ in $U$, that is, $d_{Y}(\phi, \psi) \equiv 0$ in $U$, for then $\phi$ is harmonic in each $U$ and hence in all of $X$. According to Theorem 1 (b) the function $d_{Y}(\phi, \psi) \in W_{0}^{1,2}(U)$ is subharmonic in $U \backslash F$, and consequently in all of $U$ by the known analogue of (a) for subharmonic functions 2 It therefore follows from [EF, Proposition 5.3 (i)] (as noted in the third paragraph following Theorem 1 above) that $d_{Y}(\phi, \psi)=0$, and so $\phi=\psi$ is indeed harmonic in $U$.

Ad (b). We may assume that $\phi_{n} \rightarrow \phi$ uniformly and that $\sup _{n} E\left(\phi_{n}\right)<\infty$, hence $E(\phi)<\infty$ by $[\mathrm{EF}$, Lemma 9.1] (which does not require that $Y$ be locally compact). With $U$ and $\psi$ as in the proof of (a) the subharmonic functions $d_{Y}\left(\phi_{n}, \psi\right)$ converge uniformly to $d_{Y}(\phi, \psi)$ because $d_{Y}\left(\phi_{n}, \phi\right) \rightarrow 0$ uniformly. It follows that $d_{Y}(\phi, \psi) \in W_{0}^{1,2}(U)$ is subharmonic in $U$ (cf. [EF. Definition 2.2]), and so $\phi=\psi$ is harmonic in $U$, as above.

Returning to Theorem 2, Part (b) was obtained (along with its converse) for the particular case $\operatorname{dim} X=1$ in [EF, Proposition 12.4] as an application of Jensen's inequality on $Y$ [EF, Proposition 12.3].

For smooth maps between smooth Riemannian manifolds without boundary (no curvature requirement) the pull-back property (b) was shown by Ishihara [Ish to characterize harmonic maps. This was extended in [EF, Theorem 12.1] to continuous maps of locally finite energy from the present polyhedral domains into Riemannian manifolds. Here is yet another case of Ishihara's characterization (proven in Section 6):

Proposition 1. Let $Y$ be a simply connected complete Riemannian polyhedron of dimension $\leq 2$ with simplexwise smooth Riemannian metric $h$ and nonpositive

\footnotetext{
${ }^{2}$ A function $u \in W_{\text {loc }}^{1,2}(X)$, subharmonic off some closed polar set $F \subset X$, can be redefined on $F$ so as to become subharmonic in all of $X$. Indeed, for any function $\lambda \in W_{0}^{1,2}(X)$ we have $\lambda_{\mid X \backslash F} \in W_{0}^{1,2}(X \backslash F)$ according to the "spectral synthesis theorem" of Beurling and Deny (cf. [De, pp. 168-172]), and hence

$$
\int_{X}\langle\nabla u, \nabla \lambda\rangle d \mu=\int_{X \backslash F}\langle\nabla u, \nabla \lambda\rangle d \mu \leq 0 .
$$

Similarly, $F$ is removable for harmonic functions (within the class $W_{\text {loc }}^{1,2}(X)$ ). It is well known that, conversely, a closed set $F \subset X$ is polar if $F$ is removable within $W_{\text {loc }}^{1,2}(X)$ for harmonic, resp. subharmonic, functions $u$. (After an easy reduction to the case where $X$ has a Green kernel, take for $u$ the swept-out (balayé) on $F$ of a bounded Green potential of a positive measure with continuous density $>0$.)
} 
Alexandrov curvature. A continuous map $\phi \in W_{\mathrm{loc}}^{1,2}(X, Y)$ is harmonic if and only if it pulls germs of continuous convex functions in $Y$ back to germs of subharmonic functions in $X$.

Theorem 3 below is a companion to Theorem 1 for targets $Y$ of upper bounded curvature, now assuming for the existence and continuity part (a) that $Y$ is locally compact. For a variant proof of the existence (and uniqueness) of the variational solution in Theorem 3 (a) without requiring local compactness of $Y$ see [F4], based on [Se2 (unpublished). The uniqueness part (b) of Theorem 3 is an extension of the corresponding result for maps between Riemannian manifolds, due to Jäger and Kaul [JK].

For the proof of that extension (given in Section 8) we prepare Lemma 2 below, providing a kind of complement to Reshetnyak's characterization by quadrilateral comparison, now for upper bounded curvature. In the standard unit sphere $S^{2}$ in $\mathbb{R}^{3}$ an (ordered) quadrilateral $P Q R S$ with perimeter $<2 \pi$ (hence with sides and diagonals of length $<\pi$ ) is said to be convex if the geodesic segments $P Q, Q R, R S$, and $S P$ form a subdivision of the boundary of some geodesically convex subset of $S^{2}$. A trapezoid in $S^{2}$ is understood here as a convex quadrilateral $P Q R S$ in $S^{2}$ such that $P Q R S$ is mapped onto $S R Q P$ under reflection in some great circle in $S^{2}$. Equivalently, the diagonals have the same length: $P R=Q S$, and so have two opposite sides: $P Q=R S$; this implies indeed, by convexity, that $Q$ and $R$ are in the same hemisphere bounded by the great circle through $P$ and $S$ (when e.g. $P \neq S$ ) ; and the triangles $P Q S$ and $S R P$ are congruent, so that the reflection of $S^{2}$ that interchanges $P$ and $S$ likewise interchanges $Q$ and $R$.

For brevity we shall denote by $a \diamond b$ the "cosine mean" of two numbers $a, b \in[0, \pi]$, defined as the unique number in $[0, \pi]$ satisfying

$$
\cos (a \diamond b)=\frac{1}{2}(\cos a+\cos b)
$$

Since cos is concave on $[0, \pi / 2]$ and decreasing on $[0, \pi]$, we have

$$
\begin{array}{ll}
a \diamond b \geq \frac{1}{2}(a+b) & \text { for } 0 \leq a, b \leq \frac{1}{2} \pi \\
a \diamond b \leq \max \{a, b\} & \text { for } 0 \leq a, b \leq \pi
\end{array}
$$

When $P Q R S$ denotes a quadrilateral in a geodesic space $Y$ of curvature $\leq 1$ (for simplicity), we say that a trapezoid $\widetilde{P} \widetilde{Q} \widetilde{R} \widetilde{S}$ in $S^{2}$ is a comparison trapezoid for $P Q R S$ if $P Q, R S \leq \pi / 2$ and

$$
\begin{array}{ll}
\widetilde{P} \widetilde{S}=P S, & \widetilde{Q} \widetilde{R}=Q R, \\
\widetilde{P} \widetilde{Q}=\widetilde{R} \widetilde{S}=P Q \diamond R S, & \widetilde{P} \widetilde{R}=\widetilde{Q} \widetilde{S} \geq P R \diamond Q S .
\end{array}
$$

Lemma 2. Let $\left(Y, d_{Y}\right)$ be a complete geodesic space of curvature $\leq 1$, and $B=$ $B_{Y}(q, R)$ a closed, geodesically convex ball in $Y$ of radius $R<\pi / 2$, satisfying bipoint uniqueness. Let $P Q R S$ be a quadrilateral in $B$ such that $P Q \leq \pi / 2, R S \leq \pi / 2$, and

$$
2(P Q \diamond R S)+Q R+P S<2 \pi
$$

(a) There exists a comparison trapezoid for PQRS in $S^{2}$. 
(b) For any comparison trapezoid $\widetilde{P} \widetilde{Q} \widetilde{R} \widetilde{S}$ for $P Q R S$ in $S^{2}$, and any two numbers $\lambda, \lambda^{\prime} \in[0,1]$, define, by abuse of notation, the following points of $Y$ :

$$
\begin{array}{ll}
P_{\lambda}=(1-\lambda) P+\lambda S, & Q_{\lambda^{\prime}}=\left(1-\lambda^{\prime}\right) Q+\lambda^{\prime} R, \\
S_{\lambda}=(1-\lambda) S+\lambda P, & R_{\lambda^{\prime}}=\left(1-\lambda^{\prime}\right) R+\lambda^{\prime} Q,
\end{array}
$$

and similarly the points $\widetilde{P}_{\lambda}, \widetilde{Q}_{\lambda^{\prime}}, \widetilde{R}_{\lambda^{\prime}}, \widetilde{S}_{\lambda}$ in $S^{2}$. Then

$$
P_{\lambda} Q_{\lambda^{\prime}} \diamond S_{\lambda} R_{\lambda^{\prime}} \leq \widetilde{P}_{\lambda} \widetilde{Q}_{\lambda^{\prime}}=\widetilde{S}_{\lambda} \widetilde{R}_{\lambda^{\prime}}
$$

Here $(1-\lambda) P+\lambda S$, say, denotes the point $P_{\lambda}$ of the geodesic segment joining $P$ and $S$ for which $P P_{\lambda}=\lambda P S$. Concerning bipoint uniqueness, see the paragraph following Theorem 3 below.

The proof of the lemma, given in Section 7, uses for (a) Reshetnyak's characterization of curvature $\leq 1$ by quadrilateral comparison $[\mathrm{R}]$; furthermore, the wellknown fact that, given 6 numbers $a_{i j} \in[0, \pi], 1 \leq i<j \leq 4$, there exist on the unit sphere $S^{3}$ in $\mathbb{R}^{4}$ four points $P_{i}, i=1,2,3,4$, with the distances $d_{S^{3}}\left(P_{i}, P_{j}\right)=a_{i j}$, if and only if the symmetric $4 \times 4$ matrix $A$ with entries $\cos a_{i j}$ above the diagonal and entries 1 in the diagonal is positive definite or semidefinite; furthermore, $S^{3}$ can be replaced by $S^{2}$ here if and only if $\operatorname{rank} A<4$. See $[\mathrm{Sbg}$. Part (b) is elementary (spherical trigonometry).

Theorem 3. Let $\left(Y, d_{Y}\right)$ be a complete geodesic space of curvature $\leq K$ for some constant $K>0$, and let $B=B_{Y}(q, R)$ be a closed, geodesically convex ball in $Y$ of radius $R<\pi /(2 \sqrt{K})$, satisfying bipoint uniqueness.

(a) If $Y$ is locally compact, or if $R<\pi /(4 \sqrt{K})$, then, for any map $\psi \in$ $W^{1,2}(X, B)$, the unique map $\phi$ of least energy in $W_{\psi}^{1,2}(X, B)(c f .(2.1))$ is Hölder continuous in $X \backslash b X$, and continuous at any point of $b X$ at which $\operatorname{tr}_{b X} \psi$ is continuous.

(b) For any two continuous maps $\phi_{0}, \phi_{1}: X \rightarrow B$ which are harmonic in $X \backslash b X$ the continuous function $\theta: X \rightarrow \mathbb{R}$ defined by

$$
\theta=\theta\left(\phi_{0}, \phi_{1}\right)=\frac{\left(1-\cos \left[\sqrt{K} d_{Y}\left(\phi_{0}, \phi_{1}\right)\right]\right) / K}{\cos \left[\sqrt{K} d_{Y}\left(\phi_{0}, q\right)\right] \cos \left[\sqrt{K} d_{Y}\left(\phi_{1}, q\right)\right]} \geq 0
$$

attains its maximum on $b X$. In particular, if $\phi_{0}=\phi_{1}$ on $b X$, then $\phi_{0}=\phi_{1}$ on $X$.

With $Y$ as stated, a convex set $V \subset Y$ of diameter $<\pi / \sqrt{K}$ is said to satisfy bipoint uniqueness if there is only one (minimizing) geodesic segment in $Y$ joining two points of $V$, and if that segment varies continuously with its endpoints (in the uniform topology on curves). Since geodesics in $V$ have no conjugate points AB1. Theorem 3], this definition agrees with the more restrictive one given by Alexander and Bishop in $\mathrm{AB} 2$, where it is also shown that the continuous dependence is automatic if $Y$ is locally compact. As shown by Alexandrov [A1], A2], $V$ satisfies bipoint uniqueness if and only if every geodesic triangle with vertices in $V$ and with perimeter $<2 \pi / \sqrt{K}$ admits triangle comparison; then the distance function $d_{Y}(\cdot, q)$ is convex in $V$ for fixed $q \in V$, and hence every ball in $V$ is convex. A convex ball $B$ (open or closed) in $Y$ of radius $R<\pi /(2 \sqrt{K})$ satisfies bipoint uniqueness if and only if $B$ satisfies radial uniqueness with respect to the center $q$ of $B$, i.e., the particular case of bipoint uniqueness where one of the above two points of $V=B$ is $q$ (kept fixed) [AB2, Theorem 4.3 and Proposition 4.2]. Bipoint 
uniqueness of $B$ is necessary for (b) even in the case where $X=[0,1]$, the harmonic maps $X \rightarrow B$ being then the geodesic segments in $B$.

Concerning (a), a map of least energy in $W_{\psi}^{1,2}(X, B)$ is called a variational solution to the Dirichlet problem in question. We prove existence of a variational solution when $Y$ is locally compact, and hence $B$ is compact, by the Hopf-Rinow theorem (cf. e.g. [EF, p. 28]): Denote

$$
E_{0}=\inf \left\{E(\phi): \phi \in W_{\psi}^{1,2}(X, B)\right\},
$$

and choose a minimizing sequence $\left(\phi_{n}\right)$. Then $\left(\phi_{n}\right)$ subconverges in $L^{2}(X, B)$ to a map $\phi \in W^{1,2}(X, B)$ with energy $E(\phi)=E_{0}$ [EF, Lemmas 9.2, 9.1]; and $\operatorname{tr}_{b X} \phi=$ $\operatorname{tr}_{b X} \psi$, by Lemma 1 (a). Thus $\phi \in W_{\psi}^{1,2}(X, B)$ is a variational solution. As such, $\phi$ is, in particular, locally $E$-minimizing in $X \backslash b X$, and hence Hölder continuous there (after being redefined uniquely on a $\mu$-nullset in $X \backslash b X$ ), at least if either $Y$ is locally compact or $R<\pi /(4 \sqrt{K})$ [F3, Theorem 2], extending [EF, Theorem 10.2]; consequently, $\phi$ is harmonic in $X \backslash b X$. Continuity of $\phi$ up to the boundary $b X$ (at any point of $b X$ at which $\psi_{\mid b X}$ is continuous) will be established in Section 8 , assuming again that $Y$ is locally compact or that $R<\pi /(4 \sqrt{K})$ (rather than just $R<\pi /(2 \sqrt{K}))$, interior continuity of $\phi$ being used. If $\phi$ is not continuous in $X \backslash b X$, our proof of (a) still shows that

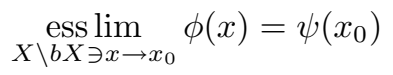

for any $x_{0} \in b X$ at which $\operatorname{tr}_{\mid b X} \psi$ is continuous.

Like in Jäger-Kaul [JK], Theorem 3 (b) will be established by proving that, if $\phi_{0}, \phi_{1} \in W_{\text {loc }}^{1,2}(X, B)$ are harmonic in $X \backslash b X$, then $\theta\left(\phi_{0}, \phi_{1}\right)$ is a local weak subsolution in $X \backslash b X$ to the linear elliptic operator $\mathcal{L}$ of divergence type defined by

$$
\mathcal{L}=\operatorname{div}(a \nabla)
$$

where $a=a\left(\phi_{0}, \phi_{1}\right)=\cos \left[\sqrt{K} d_{Y}\left(\phi_{0}, q\right)\right] \cos \left[\sqrt{K} d_{Y}\left(\phi_{1}, q\right)\right]$ is a bounded function of class $W^{1,2}(X)$. Once that has been proved (in Section 8, by use of Lemma 2) it remains to extend Hopf's maximum principle for the operator $\mathcal{L}$ to the present setting of a polyhedral domain $X$. For this, the function $a$ may even be replaced by any bounded measurable function $a>0$, bounded away from 0 . The local subsolutions to $\mathcal{L}$ are then nothing but the weakly subharmonic functions for a new Brelot harmonic sheaf and Dirichlet structure on $X$, obtained simply by replacing the Riemannian volume element $d \mu=\sqrt{\operatorname{det} g} d x^{1} \cdots d x^{m}$ on $X$ by $a d \mu$, and hence the Dirichlet form $\int\langle\nabla u, \nabla v\rangle d \mu$ by $\int\langle\nabla u, \nabla v\rangle a d \mu$. This substitution causes no problems whatsoever, and so the maximum principles in [EF, Proposition 5.3] carry over and replace the Hopf maximum principle used in $[\mathrm{JK}$ in the manifold case. See Hervé [Her] for the case of a bounded domain $X$ in $\mathbb{R}^{m}$.

For any two variational solutions $\phi_{0}, \phi_{1}$ to the Dirichlet problem the distance function $d_{Y}\left(\phi_{0}, \phi_{1}\right)$ is of class $W^{1,2}(X)$, by [EF, Corollaries 9.1, 9.2], hence so is $\theta\left(\phi_{0}, \phi_{1}\right)$. It therefore follows from Part (i) of [EF, Proposition 5.3] (applied to the operator $\mathcal{L}$ in place of the weak Laplacian, and to $X \backslash b X$ in place of $X$ ) that $\theta\left(\phi_{0}, \phi_{1}\right) \leq 0$ a.e. in $X \backslash b X$, and so $\theta\left(\phi_{0}, \phi_{1}\right)=0$ there; i.e., $\phi_{0}=\phi_{1}$ in $X \backslash b X$ and hence a.e. in $X$. This establishes uniqueness of the variational solution.

If $\operatorname{tr}_{b X} \psi$ is continuous, and if $\phi_{0}, \phi_{1}$ are two continuous maps of class $W_{\psi}^{1,2}(X, B)$ which are harmonic in $X \backslash b X$, then Part (ii) of [EF, Proposition 5.3] shows that 
the continuous function $\theta\left(\phi_{0}, \phi_{1}\right)$ on $X$ is $\leq 0$ in $X \backslash b X$, hence $\phi_{0}=\phi_{1}$ in $X \backslash b X$, and in all of $X$, by continuity. Thus the classical-style Dirichlet problem of finding a continuous map $\phi \in W_{\psi}^{1,2}(X, B)$ with $\phi$ harmonic in $X \backslash b X$, is uniquely solvable and well posed for maps into the ball $B=B_{Y}(q, R)$.

The above additional hypothesis that $Y$ be locally compact is actually not needed for the existence (and uniqueness) of the variational solution. This was shown (in the typical case where the domain of the maps is a Lipschitz Riemannian domain) by [Se2] (unpublished) 3

For the particular case of a smoothly bounded Riemannian domain it was shown in [Se2 that the variational solution $\phi$ is even Lipschitz continuous in $X \backslash b X]$ this fails for polyhedral $X$, even when $Y=\mathbb{R}$; see e.g. [EF Example 6.1].

The hypothesis $R<\pi /(2 \sqrt{K})$ in Theorem 3 cannot be replaced by $R \leq \pi /(2 \sqrt{K})$, not even for maps between Riemannian manifolds. Thus interior regularity may break down, as shown by an example due to Hildebrandt, Kaul, and Widman HKW, §6]; cf. [EF, Example 12.3]. Uniqueness of the variational solution will of course break down even in the simplest case of geodesics on a closed hemisphere.

Maps into a Riemannian manifold. In this subsection the Riemannian metric $g$ on each $m$-simplex of the $m$-dimensional admissible polyhedron $X$ is allowed to be just bounded and measurable (rather than smooth), and as always nondegenerate, i.e., with elliptic bounds (cf. [EF, eq. (4.1)]). That suffices for the definition and properties of a natural covariant concept of energy of maps into manifolds as in [EF, Definition 9.2]; see Definition 2 below. For simplicity of enunciations we suppose here that $X$ is compact; but at first we do not insist that $X$ have a boundary $b X \neq \varnothing$. Let $(N, h)$ be an $n$-dimensional Riemannian $C^{1}$-manifold without boundary, having a countable base for its topology, and endowed with a countable atlas. In particular, $N$ is locally compact.

Definition 2. A map $\phi$ of $(X, g) \rightarrow(N, h)$ has finite energy if and only if

(i) $\phi$ can be redefined on a $\mu$-nullset so as to become quasicontinuous; and

(ii) the components $\phi^{1}, \ldots, \phi^{n}$ of $\phi$ in each chart $V \rightarrow \mathbb{R}^{n}$ are of class $W^{1,2}$ on the quasiopen set $\phi^{-1}(V)$; and

(iii) the energy density $e(\phi)$ of $\phi$, defined $\mu$-a.e. in each of the sets $\phi^{-1}(V)$ covering $X$ by

$$
e(\phi)=\operatorname{trace}_{g} \phi^{*} h=\left(h_{\alpha \beta} \circ \phi\right)\left\langle\nabla \phi^{\alpha}, \nabla \phi^{\beta}\right\rangle
$$

(summed over $\alpha, \beta=1, \ldots, n)$, is integrable over $(X, \mu)$.

The energy of $\phi$ is then defined by $E(\phi)=\int e(\phi) d \mu$.

For the terms quasiopen and quasicontinuous see $[\mathrm{EF}$. Definitions 7.2, 7.3], where the capacity is associated with the Dirichlet form $\int_{X}|\nabla u|^{2} d \mu, u \in L_{0}^{1,2}(X)$ (see EF p. 21 and Proposition 7.3]), possibly applied locally; cf. [EF, Remark 7.2].

Note that the above pre-images $\phi^{-1}(V)$ in (ii) and (iii) are quasiopen in view of (i); we refer to Kilpeläinen and Malý [KM] for the Sobolev space $W^{1,2}(U)$ on a

\footnotetext{
${ }^{3} \mathrm{In}[\mathrm{Se} 2$, and hence (sic!) in $[\mathrm{EF}$, the inequality reproduced in $[\mathrm{EF}$, eq. (11.15)] is unfortunately misstated, the "hat" over $\phi_{1 / 2}$ being missing (thereby invalidating the inequality, even in the case of geodesics on a standard 2-sphere). The proof in Se2 pertains of course to the correct version of the inequality and is completed and extended to polyhedral domains in [F4].

${ }^{4}$ Continuity of $\phi$ up to the boundary, though mentioned in [EF, p. 210] without justification, is not claimed in [Se2].
} 
quasiopen set $U \subset \mathbb{R}^{m}$ and the gradient operator $\nabla$ on it, equally applicable in the present setting, where $U \subset X$. See [EF, text following Definition 7.4].

If $\phi(X)$ has compact closure in $N$, then (iii) is a consequence of (i) and (ii) because $\phi(X)$ is covered by finitely many coordinate patches $V$. If $\phi$ is even continuous (or if $\phi(X)$ is contained in a single coordinate patch $V$, as in Theorems 4 and 5 below), then $W^{1,2}\left(\phi^{-1}(V)\right)$ becomes the usual Sobolev $(1,2)$-space on $\phi^{-1}(V)=X$.

The above definition is independent of the choice of countable atlas on $N$ EF text following Definition 9.2].

Proposition 2. Given a map $\phi:(X, g) \rightarrow(N, h)$ with $g$ simplexwise smooth, suppose either that

(a) $\phi(X)$ has compact closure in $N$ (e.g. $\phi$ is continuous); or that

(b) $N$ is simply connected, complete, and has nonpositive sectional curvature.

Then the energy of $\phi$ in the sense of Definition 1 equals the energy of $\phi$ in the sense of Definition 2, multiplied by the constant factor $c_{m}$ from (1.4); and in case of finite energy, the energy densities of $\phi$ under the two definitions are similarly related.

In case (a), this follows from [EF, Theorem 9.2]. Case (b) is derived from (a) in Section 9 .

In the rest of this subsection, $g$ may again be just bounded measurable, and Definition 2 of energy is used. In combination with Proposition 2 the following lemma serves as a key to transferring the existence theory from [EF, Chapter 11] to the present setting of maps into manifolds; cf. Remark 1 below:

Lemma 3. With g just bounded measurable, let $(N, h)$ be simply connected complete with nonpositive sectional curvature, and let $\phi_{0}, \phi_{1} \in W^{1,2}(X, N)$ (Definition 2). For $t \in[0,1]$ the map $\phi_{t}: X \rightarrow N$ defined (by abuse of notation) by $\phi_{t}(x)=$ $(1-t) \phi_{0}(x)+t \phi_{1}(x), x \in X$, satisfies

$$
E\left(\phi_{t}\right) \leq(1-t) E\left(\phi_{0}\right)+t E\left(\phi_{1}\right)-t(1-t) \int_{X}\left|\nabla d_{N}\left(\phi_{0}, \phi_{1}\right)\right|^{2} d \mu .
$$

A corresponding inequality holds $\mu$-a.e. in $X$ for the energy densities $e(\cdot)$ in place of the energies $E(\cdot)$ of $\phi_{t}, \phi_{0}, \phi_{1}$, omitting the integration. For the proof see Section 10.

For a finite energy map of $(X, g)$ into a complete Riemannian manifold $(N, h)$ the concept of trace on a compact subset of $b X$ does not involve the Riemannian metric $g$ on $X$, and therefore remains in force when $g$ is just bounded measurable. Lemma 1 remains valid because the assertions are not affected when $g$ is replaced by a Euclidean (hence simplexwise smooth) Riemannian metric $g^{e}$ on $X$.

Here is a companion to Theorem 1 in the present setting with $X$ compact and $b X \neq \varnothing$ (using now Definition 2 of energy, $g$ being just bounded measurable). Recall the definition $(2.1)$ of $W_{\psi}^{1,2}(X, N)$.

Theorem 4. Let $(N, h)$ be a simply connected complete smooth Riemannian manifold (without boundary) of nonpositive sectional curvature.

(a) For any map $\psi \in W^{1,2}(X, N)$ the unique map $\phi$ of least energy in $W_{\psi}^{1,2}(X, N)$ is continuous at any point of $b X$ at which $\operatorname{tr}_{b X} \psi$ is continuous.

(b) For any two continuous maps $\phi_{0}, \phi_{1}: X \rightarrow N$ which are harmonic in $X \backslash b X$ the distance function $d_{Y}\left(\phi_{0}, \phi_{1}\right) \in W^{1,2}(X)$ is subharmonic in $X \backslash b X$, and hence attains its maximum on $b X$. In particular, if $\phi_{0}=\phi_{1}$ on $b X$, then $\phi_{0}=\phi_{1}$ on $X$. 
The existence and uniqueness of the variational solution $\phi$ in (a) is contained in EF Theorem 11.3] when $g$ is simplexwise smooth, in view of Proposition 2 (b); the proof carries over to the present case where $g$ is just bounded measurable, in view of Lemma 3 above and [EF, Remark 9.6]. Hölder continuity of $\phi$ in $X \backslash b X$ follows from [F5 Proposition 2], [F3, Theorem 3]. Continuity up to the boundary is proved just like in the case of Theorem 1 (a); see Section 4.

The proof of (b) is simply carried over from the case settled by Jäger and Kaul JK], where $X$ is a Riemannian manifold with boundary. If $\operatorname{tr}_{b X} \psi$ is continuous, it follows from (a) and (b) that $\phi$ is continuous in all of $X$, and is thus the unique solution to the classical-style Dirichlet problem in $X$ with continuous boundary $\operatorname{map} \operatorname{tr}_{b X} \psi$.

Without assuming $X$ compact and $b X \neq \varnothing$ it still holds of course that $d_{N}\left(\phi_{0}, \phi_{1}\right)$ is subharmonic when $\phi_{0}, \phi_{1}: X \rightarrow N$ are harmonic. Here is a short, alternative proof of that: Since $\phi_{0}$ and $\phi_{1}$ are weakly harmonic, by [EF] Theorem 12.1], so is clearly $\phi_{0} \times \phi_{1}: X \rightarrow N \times N$. Because $N$ is globally squared-convex (cf. e.g. EF Chapter 2]), the intrinsic distance $d_{N}$ is convex on $N \times N$, and its pull-back $d_{N}\left(\phi_{0}, \phi_{1}\right)$ under $\phi_{0} \times \phi_{1}$ is therefore indeed subharmonic according to Ishihara's characterization of harmonic maps between (smooth) Riemannian manifolds [Ish], extended in [EF, Theorem 12.1] to the present setting of maps with polyhedral domain $(X, g), g$ being just measurable and bounded on each top-dimensional simplex.

For the case where $(X, g)$ is a Riemannian manifold with boundary, Theorem 4 was obtained, along with further regularity results, by Hildebrandt, Kaul, and Widman [HKW] and by Giaquinta and Hildebrandt [GH]. The same applies to the following companion to Theorem 3 for the case where $(N, h)$ has just upper bounded curvature (and $g$ is just measurable and locally bounded).

Theorem 5. Let $(N, h)$ be a complete smooth Riemannian manifold (without boundary) of sectional curvature $\leq K$ for some constant $K>0$, and let $B=$ $B_{N}(q, R)$ be a closed, geodesically convex Riemannian ball of radius $R<\pi /(2 \sqrt{K})$, lying within normal range from each of its points (i.e., $B$ shall not meet the cut locus of any of its points).

(a) For any map $\psi \in W^{1,2}(X, B)$ the unique map $\phi$ of least energy in $W_{\psi}^{1,2}(X, B)$ is continuous at any point of $b X$ at which $\operatorname{tr}_{b X} \psi$ is continuous.

(b) For any two continuous maps $\phi_{0}, \phi_{1}: X \rightarrow B$ which are harmonic in $X \backslash b X$ the continuous function $\theta=\theta\left(\phi_{0}, \phi_{1}\right) \geq 0$ defined in (2.6) attains its maximum on $b X$. In particular, if $\phi_{0}=\phi_{1}$ on $b X$, then $\phi_{0}=\phi_{1}$ on $X$.

Under the remaining hypotheses on $N$ and $B$ the cut locus condition is fulfilled if and only if $B$ satisfies bipoint uniqueness, or equivalently: radial uniqueness, AB2, $\S 4]$ (see also the paragraphs following Theorems 2 and 4 in [F3]).

Note that $B$ is compact, by the Hopf-Rinow theorem. The existence of a variational solution $\phi$ in (a) is therefore established as above concerning Theorem 3 (a) when $g$ is simplexwise smooth, in view of [EF, Theorem 9.2] (or Proposition 2 above); the proof carries over to the present case where $g$ is just bounded measurable, in view of [EF Remark 9.6].

Hölder continuity of $\phi$ in $X \backslash b X$ follows from [F5, Proposition 2], [F3, Theorem 4]. Continuity up to the boundary is proved just like in the case of Theorem 3 (a) in Section 8. 
The uniqueness proof in [JK] for the case of a manifold domain $X$ carries over to the present setting in view of the properties of the subsolutions to $\mathcal{L}$ described in the paragraph containing (2.7) above; this establishes (b).

Remark 2. Theorem 2 and Corollaries 1 and 2 above carry over rightaway to the present setting with $g$ just bounded measurable (but with manifold target $(N, h)$ ), using now Definition 2, and invoking [EF, Remark 9.6]. Like the existence and uniqueness of the variational solution to the Dirichlet problem, other results in EF Chapter 11] have companions in the present setting. Suppose that $X$ and $N$ are compact and that $(N, h)$ has nonpositive sectional curvature:

(1) Every homotopy class $\mathcal{H}$ of continuous maps $X \rightarrow N$ contains maps of least energy, and any such map is Hölder continuous. If two E-minimizers in $\mathcal{H}$ agree at a point of $X$, then they are identical. See [EF, Theorem 11.1 and Corollary 11.1].

(2) Suppose that $b X \neq \varnothing$. Given a continuous map $\psi: X \rightarrow N$, let $\mathcal{C}_{\psi}(X, N)$

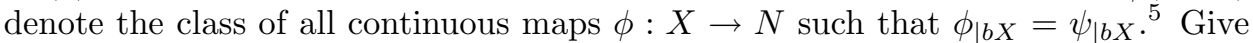
$\mathcal{C}_{\psi}(X, N)$ the topology of uniform convergence on $X$. Every homotopy class $\mathcal{H}$ in $\mathcal{C}_{\psi}(X, N)$ (i.e., every connectivity component of $\mathcal{C}_{\psi}(X, N)$ ) has a unique element $\phi$ of least energy, and $\phi$ is Hölder continuous in $X \backslash b X$. See [EF, Theorem 11.2], [F3, Theorem 3].

In view of Lemma 3 these assertions are obtained from the proofs of the quoted results from $\mathrm{EF}$ (where $g$ is required to be simplexwise smooth). See also EF Remark 11.4], proven in [F5].

\section{Proof of Lemma 1}

It suffices to consider the case where $X$ is embedded in some $\mathbb{R}^{N}$, with every simplex affinely embedded [EF, Remark 4.1]; furthermore, $g=g^{e}$ (a Euclidean Riemannian metric), and $d_{X}=d_{X}^{e}$ (the Euclidian Riemannian distance on $X$ ); cf. [EF pp. 49-53].

Proof of (a). We have $E(\phi) \leq \liminf _{n} E\left(\phi_{n}\right)<\infty$ according to EF Lemma 9.1], generalizing $[\mathrm{KS}$, Theorem 1.6.1] and valid without requiring that $Y$ be locally compact. For every $(m-1)$-simplex $\sigma$ of $b X$ with associated $m$-simplex $s \supset \sigma$ it follows by [KS, Theorem 1.12.2] (applied to the Lipschitz Riemannian domain $s^{\circ}$, the relative interior of $s$ ) that $\operatorname{tr}_{\sigma} \phi_{n} \rightarrow \operatorname{tr}_{\sigma} \phi$ in $L^{2}(\sigma, Y)$; so, indeed, $\operatorname{tr}_{b X} \phi_{n} \rightarrow$ $\operatorname{tr}_{b X} \phi$ in $L^{2}(b X, Y)$.

Proof of (b). Write $d_{Y}(\phi, \psi)=u$, and suppose first that $\operatorname{tr}_{\Gamma} u=0$. The proof of [KS, (1.12vi)], applied to the above Lipschitz Riemannian domain $s^{\circ}$ with an $(m-1)$-face $\sigma$ in $b X$, extends right away when the entire boundary $b s$ is replaced by $\gamma:=\Gamma \cap \sigma$; cf. [KS, (1.12ii)]. When applied to the function $u: s^{\circ} \rightarrow \mathbb{R}$ with $\operatorname{tr}_{\gamma} u=0$ this leads $\operatorname{td}^{6}$

$$
\int_{s(\gamma, \rho t)} u^{2} d \mu \leq C t^{2} \int_{s(\gamma, t)}|\nabla u|^{2} d \mu
$$

\footnotetext{
${ }^{5}$ The proof of [EF, Theorem 11.2] covers the stronger assertion in which $\mathcal{C}_{\psi}(X, Y)$ is defined as $\mathcal{C}_{\psi}(X, N)$ above, i.e., without requiring that its members have finite energy.

${ }^{6}$ The proof of [KS (1.12ii)] justifies the choice of $s(\gamma, t)$ as domain of integration on the right of (3.1), instead of the larger domain $\left\{x \in s: d_{X}(x, \partial s) \leq t\right\}$.
} 
where $s(\gamma, t):=\left\{x \in s: d_{X}(x, \gamma) \leq t\right\}$ and $\left.\rho \in\right] 0,1[$ is a certain Lipschitz constant, while $C$ stands for various constants. From [KS (1.12vii)] we likewise obtain for the maps $\phi, \psi \in W^{1,2}\left(s^{\circ}, Y\right)$,

$$
\begin{aligned}
& \left(\int_{\gamma} d_{Y}^{2}\left(\operatorname{tr}_{\gamma} \phi, \operatorname{tr}_{\gamma} \psi\right) d \mathcal{H}_{m-1}\right)^{1 / 2} \\
& \quad \leq C t^{1 / 2}\left(E(\phi)^{1 / 2}+E(\psi)^{1 / 2}\right)+C t^{-1 / 2}\left(\int_{s(\gamma, t)} u^{2} d \mu\right)^{1 / 2}
\end{aligned}
$$

which converges to 0 as $t \rightarrow 0$, by (3.1). Consequently, $\operatorname{tr}_{\gamma} \phi=\operatorname{tr}_{\gamma} \psi \mu$-a.e. in $\gamma$, and hence $\operatorname{tr}_{\Gamma} \phi=\operatorname{tr}_{\Gamma} \psi \mu$-a.e. in $\Gamma$, by varying $\sigma$.

Conversely, suppose that $\operatorname{tr}_{\Gamma} \phi=\operatorname{tr}_{\Gamma} \psi$. With $\sigma, s$, and $\gamma=\Gamma \cap \sigma$ as above, the proof of [KS, (1.12ix)] shows that (since $\operatorname{tr}_{\gamma} \phi=\operatorname{tr}_{\gamma} \psi$ )

$$
\lim _{t \rightarrow 0} t^{-2} \int_{s(\gamma, t)} d_{Y}^{2}(\phi, \psi) d \mu=0 .
$$

We show that (3.2) remains valid when $\gamma$ is replaced by $\Gamma$ and $s$ by $X$; i.e., $\operatorname{tr}_{\Gamma} \phi=$ $\operatorname{tr}_{\Gamma} \psi$ implies

$$
\lim _{t \rightarrow 0} t^{-2} \int_{X(\Gamma, t)} d_{Y}^{2}(\phi, \psi) d \mu=0,
$$

where $X(\Gamma, t):=\left\{x \in X: d_{X}(x, \Gamma) \leq t\right\}$. For that it suffices to verify (3.2) with $s(\gamma, t)$ replaced by $s^{\prime}(\gamma, t):=\left\{x \in s^{\prime}: d_{X}(x, \gamma) \leq t\right\}$ for any $m$-simplex $s^{\prime} \neq s$ of $X$; this leads in fact to (3.3) by summation because the sets $s^{\prime}(\gamma, t)$ cover $X(\Gamma, t)$ when $\sigma$ and $s^{\prime}$ vary.

Because $X$ is admissible (in particular connected) we may join $s$ to $s^{\prime}$ by a chain $A=s_{0} \cup s_{1} \cup \cdots \cup s_{p}$ of $p+1$ distinct $m$-simplexes $s_{i}$ of $X$ such that $s_{0}=s$, $s_{p}=s^{\prime}$, and that for every $i \in\{1, \ldots, p\}$ we have $\operatorname{dim}\left(s_{i} \cap s_{i-1}\right)=m-1$, and $\left(s_{i} \backslash s_{i-1}\right) \cap s_{j}=\varnothing$ for $j<i-1$. We may assume that $p$ is as small as possible. Write $\sigma=\sigma_{0}$ and $s_{i} \cap s_{i-1}=\sigma_{i}(i \in\{1, \ldots, p\})$. We proceed to construct a bi-Lip bijection $\alpha: A \rightarrow s$ leaving $\sigma$ pointwise fixed.

Denote $a_{i}$ the vertex of $s_{i}$ opposite $\sigma_{i}, i \in\{0,1, \ldots, p\}$. Suppose $i \geq 1$, and write $\sigma_{i} \cap \sigma_{i-1}=\tau_{i}$; then $\operatorname{dim} \tau_{i}=m-2$. Denote $b_{i}$ the vertex of $\sigma_{i-1}$ opposite $\tau_{i}$. Choose a point $c_{i}$ between $b_{i}$ and $a_{i-1}$ on the segment joining them, and denote

$$
\beta_{i}: s_{i-1} \rightarrow \operatorname{conv}\left(\left\{b_{i}, c_{i}\right\} \cup \tau_{i}\right)
$$

the affine bijection between $m$-simplexes defined by $\beta_{i}\left(a_{i-1}\right)=c_{i}, \beta_{i}\left(b_{i}\right)=b_{i}$, and $\beta_{i}(x)=x$ for $x \in \tau_{i}$. (Here conv indicates convex hull.) Denote

$$
\beta_{i}^{\prime}: s_{i} \rightarrow \operatorname{conv}\left(\left\{a_{i-1}, c_{i}\right\} \cup \tau_{i}\right)
$$

the affine bijection between $m$-simplexes defined by $\beta_{i}^{\prime}\left(a_{i}\right)=a_{i-1}, \beta_{i}^{\prime}\left(a_{i-1}\right)=c_{i}$, and $\beta_{i}^{\prime}(x)=x$ for $x \in \tau_{i}$. Then $\beta_{i}$ and $\beta_{i}^{\prime}$ combine to a bi-Lip bijection

$$
\beta_{i} \cup \beta_{i}^{\prime}: s_{i-1} \cup s_{i} \rightarrow s_{i-1}
$$

leaving the $(m-1)$-simplex $\sigma_{i-1}=\operatorname{conv}\left(\left\{b_{i}\right\} \cup \tau_{i}\right)$ pointwise fixed. If $i \geq 2, \beta_{i} \cup \beta_{i}^{\prime}$ therefore combines with the identity map of $s_{0} \cup \cdots \cup s_{i-2}$ to a bi-Lip bijection

$$
\alpha_{i}: s_{0} \cup \cdots \cup s_{i-1} \cup s_{i} \rightarrow s_{0} \cup \cdots \cup s_{i-1}
$$


leaving the $(m-1)$-simplex $\sigma_{0}(=\sigma)$ pointwise fixed. (If $i=1$, put $\alpha_{i}=\beta_{i} \cup \beta_{i}^{\prime}$.) The map

$$
\alpha:=\alpha_{1} \circ \cdots \circ \alpha_{p}: A \rightarrow s_{0}(=s)
$$

is the desired bi-Lip bijection. Note that $\alpha$ maps $s$ affinely onto $\alpha(s)=\beta_{1}\left(s_{0}\right)=$ $\operatorname{conv}\left(\left\{c_{1}\right\} \cup \sigma\right) \subset s$, and that $\alpha(x)=\beta_{1}(x)=x$ for $x \in \sigma$. Using [KS, Lemma 1.12.1] we infer that $\operatorname{tr}_{\sigma} \phi=\operatorname{tr}_{\sigma}\left(\phi \circ \alpha^{-1}\right)$, and similarly for $\psi$.

From (3.2), with $\phi, \psi$ replaced by $\phi \circ \alpha^{-1}, \psi \circ \alpha^{-1}$, we therefore obtain, in terms of the bi-Lip constant $\|\alpha\| \geq 1$ of $\alpha$, writing $d_{Y}(\phi, \psi)=u\left(\in W^{1,2}(X)\right)$, and taking into account that $s^{\prime}=s_{p} \subset A$, that $\alpha^{-1}\left(s^{\prime}(\gamma, t)\right) \subset s(\gamma,\|\alpha\| t)$, and that $\operatorname{tr}_{\gamma}\left(\phi \circ \alpha^{-1}\right)=\operatorname{tr}_{\gamma} \phi=\operatorname{tr}_{\gamma} \psi=\operatorname{tr}_{\gamma}\left(\psi \circ \alpha^{-1}\right)$ :

$$
t^{-2} \int_{s^{\prime}(\gamma, t)} u^{2} d \mu \leq\|\alpha\|^{m+2}(\|\alpha\| t)^{-2} \int_{s(\gamma,\|\alpha\| t)}\left(u \circ \alpha^{-1}\right)^{2} d \mu \rightarrow 0
$$

as $t \rightarrow 0$. Consequently, $\operatorname{tr}_{\Gamma} \phi=\operatorname{tr}_{\Gamma} \psi$ implies (3.3), by the text following it.

Proceeding as in [KS, p. 609], we furthermore deduce from $\operatorname{tr}_{\Gamma} \phi=\operatorname{tr}_{\Gamma} \psi$ that $u \in W_{0}^{1,2}(X \backslash \Gamma)$ : Define a Lip function $\eta_{t}: X \rightarrow[0,1]$ by $\eta_{t}=0$ in $X\left(\Gamma, \frac{1}{2} t\right)$, $\eta_{t}=1$ in $X \backslash X(\Gamma, t)$, and $\eta_{t}(x)$ affine elsewhere as a function of $d_{X}(\Gamma, x)$. Then $\left|\nabla \eta_{t}\right| \leq 2 / t \mu$-a.e. It follows easily that $\eta_{t} u \rightarrow u$ in $W^{1,2}(X)$ as $t \rightarrow 0$, and so $u \in W_{0}^{1,2}(X \backslash \Gamma)$ because $\eta_{t} u \in W_{c}^{1,2}(X \backslash \Gamma)$.

Conversely, $u \in W_{0}^{1,2}(X \backslash \Gamma)$ implies $\operatorname{tr}_{\Gamma} u=0$ by (a) applied to a sequence of functions $u_{n} \in \operatorname{Lip}_{c}(X \backslash \Gamma)$ converging to $u$ in $W^{1,2}(X)$ (hence in $L^{2}(X)$ ), noting that the energies $E\left(u_{n}\right)=\int_{X}\left|\nabla u_{n}\right|^{2} d \mu$ converge, hence remain bounded, and that obviously $\operatorname{tr}_{\Gamma} u_{n}=0$.

Proof of (c). The proof will show that it suffices to require that $u \in W_{0}^{1,2}(X \backslash \sigma)$ (or equally well $u \in \operatorname{Lip}_{c}(X \backslash \sigma)$ ) for some $(m-1)$-simplex $\sigma$ of $b X$. Let $s^{\prime}$ be a given $m$-simplex of $X$, and denote $s$ the unique $m$-simplex having $\sigma$ as a face. It suffices to prove the stated inequality with $X$ replaced by $s^{\prime}$ on the left. Via a chain $A$ joining $s$ to $s^{\prime}$, and a bi-Lip bijection $\alpha: A \rightarrow s$ as in the proof of (b) above, the claim further reduces to the well-known case $X=s^{\prime}=s$ :

$$
\int_{s} u^{2} d \mu \leq C \int_{s}|\nabla u|^{2} d \mu \quad \text { for } u \in \operatorname{Lip}_{c}(X \backslash \sigma) .
$$

To establish (3.4), denote $v$ the unit vector in $\mathbb{R}^{m} \supset s$ parallel to the median in $s$ to the midpoint of $\sigma$, and denote $c$ the length of that median. Then $(\xi, t) \mapsto x:=\xi+t v$ defines a linear bijection $\theta$ of a set $S$ of the form $S=\{(\xi, t): \xi \in \sigma, t \leq \tau(\xi)\}$, with $\tau(\xi) \leq c$, onto $s$. Because $u=0$ on $\sigma$ we have

$$
u^{2}(\xi+t v) \leq\left(\int_{0}^{\tau(\xi)}|\nabla u(\xi+t v)| d t\right)^{2} \leq c \int_{0}^{\tau(\xi)}|\nabla u(\xi+t v)|^{2} d t,
$$

and that leads by integration to (3.4).

\section{Proof of Theorem 1}

Proof of (a). As explained in Section 2 (after stating the theorem) we employ a version of $\phi \in W^{1,2}(X, Y)$ such that $\phi_{\mid X \backslash b X}$ is (Hölder) continuous and that $\phi_{\mid b X}$ is a version of $\operatorname{tr}_{b X} \phi=\operatorname{tr}_{b X} \psi \in L^{2}(b X, Y)$ for which $\phi_{\mid b X}$ is continuous at a given point $x_{0} \in b X$. We shall prove that $\phi: X \rightarrow Y$ is continuous at $x_{0}$. That will be done by reduction to the case $\left(Y, d_{Y}\right)=\mathbb{R}$ of harmonic functions. 
Consider a function $f \in W^{1,2}(X)$ such that $f_{\mid b X}$ is a version of $\operatorname{tr}_{b X} f$, continuous at $x_{0}$ with $f\left(x_{0}\right)=0$. Let $u: X \backslash b X \rightarrow \mathbb{R}$ denote the unique harmonic function in $X \backslash b X$ such that $(u-f)_{\mid X \backslash b X} \in W_{0}^{1,2}(X \backslash b X)$; see [KS, §2.2] or [EF. Theorem $5.2]$ for this variational solution, drawing on the Poincaré inequality of Lemma 1 (c). Equivalently, $\operatorname{tr}_{b X} u=\operatorname{tr}_{b X} f$, in view of Lemma 1 (b). We show that

$$
u(x) \rightarrow 0 \quad \text { as } x \rightarrow x_{0}, x \in X \backslash b X .
$$

This is done somewhat in analogy with Steps 1 and 2 of the proof of [EF, Proposition 7.1] (Lipschitz functions being now replaced by $W^{1,2}$-functions):

Step 1. Suppose that $f=0$ everywhere in a neighbourhood $V$ of $x_{0}$ relative to $b X$. In a sufficiently fine triangulation of $X$, in which $x_{0}$ is a vertex, the (open) star $\operatorname{st}_{X}\left(x_{0}\right)$ is the interior of its closure $Z_{+}$, and $Z_{+} \cap b X \subset V$. By hypothesis, $\operatorname{tr}_{Z_{0}} f=0$, and hence $f_{\mid Z_{+} \backslash Z_{0}} \in W_{0}^{1,2}\left(Z_{+} \backslash Z_{0}\right)$ according to Lemma 1 (b).

Let $Z_{-}$denote another copy of $Z_{+}$, and denote by $Z$ the disjoint union of $Z_{+}$ and $Z_{-}$, though with $Z_{+} \cap b X$ and $Z_{-} \cap b X$ identified pointwise, and then denoted $Z_{0}$. (All this is of course understood via a simplicial complex which is mapped Lip-homeomorphically onto $Z_{+}$; cf. [EF, pp. 42-46].) There is a natural bijection $\tau: Z \rightarrow Z$ interchanging $Z_{+}$and $Z_{-}$and having $Z_{0}$ as its fixed-point set. Like $Z_{+}$(and hence $Z_{-}$), $Z$ is an admissible $m$-dimensional polyhedron. To see that $Z$ is indeed locally $(m-1)$-chainable, consider two $m$-simplexes $s, s^{\prime}$ of $Z$ with a common face $\sigma$. In the nontrivial case where, e.g., $s \subset Z_{+}$and $s^{\prime} \subset Z_{-}$, hence $\tau\left(s^{\prime}\right) \subset Z_{+}$, we have $\sigma \subset s \cap s^{\prime} \subset Z_{0}$, and since $Z_{+}$is admissible, $s$ can be joined to $\tau\left(s^{\prime}\right)(\supset \sigma)$ by a chain of $m$-simplexes of $Z_{+}$(containing $\left.\sigma\right)$. Because $Z_{0} \subset b Z_{+}$, $\sigma$ is a face of some $(m-1)$-simplex $t$ of $Z_{0}$; and $t$ is a face of a unique $m$-simplex $s_{+}$of $Z_{+}$, hence also of $s_{-}:=\tau\left(s_{+}\right) \subset Z_{-}$. Since $\sigma \subset t \subset s_{+} \cap s_{-}, s$ can be joined to $s_{+}$within $Z_{+}$, and $s^{\prime}$ to $s_{-}$within $Z_{-}$. Finally, $s_{+}$and $s_{-}$are already joined in the same way within $Z$ because $s_{+} \cap s_{-}=t$ has dimension $m-1$.

Now transport the given Riemannian structure $g$ from $Z_{+}$to $Z_{-}$, thereby turning $Z$ into a (compact admissible) Riemannian polyhedron $(Z, g)$, and hence a geodesic space, with intrinsic Riemannian distance denoted $d_{Z}$ [EF, Proposition 4.1].

For a function $h \in \operatorname{Lip}_{c}\left(Z_{+} \backslash Z_{0}\right)$ the $(\tau$-)antisymmetric extension of $h$ across $Z_{0}$ (likewise denoted $h$ ) is of class $\operatorname{Lip}(Z)$. Indeed, for points $x \in Z_{+}$and $y \in Z_{-}$, a geodesic segment $[x, y]$ in $Z$ has some point $z$ in common with $Z_{0}$, and since $h(z)=0$ we obtain, denoting by $C$ the Lip constant of $h: Z_{+} \rightarrow \mathbb{R}$ :

$$
\begin{aligned}
|h(x)-h(y)| & =|h(x)+h(\tau(y))| \leq|h(x)|+|h(\tau(y))| \\
& \leq C d_{Z_{+}}(x, z)+C d_{Z_{-}}(\tau(y), z) \\
& =C d_{Z}(x, z)+C d_{Z}(\tau(y), z)=C d_{Z}(x, y) .
\end{aligned}
$$

Since $\tau(z)=z$ we have indeed $d_{Z_{+}}(x, z)=d_{Z}(x, z)$. (Consider a geodesic segment $\gamma$ in $Z$ joining $x$ and $z$. Replacing the parts of $\gamma$ in $Z_{-} \backslash Z_{0}$ by their images under $\tau$, we obtain a new geodesic in $Z$ joining $x$ and $z$ and with the same length $d_{Z}(x, z)$, but lying entirely in $Z_{+}$, whence the assertion.) Likewise, $d_{Z_{-}}(\tau(y), z)=$ $d_{Z}(\tau(y), z)=d_{Z}(y, z)$, the latter again because $\tau(z)=z$.

As already noted, the given function $f \in W^{1,2}(X)$, restricted to $Z_{+}$, is of class $W_{0}^{1,2}\left(Z_{+} \backslash Z_{0}\right)$ and can therefore be approximated in $W^{1,2}\left(Z_{+}\right)$by functions $h \in \operatorname{Lip}_{c}\left(Z_{+} \backslash Z_{0}\right)$ as considered above. The antisymmetric extension of $f$ (likewise denoted $f$ ) is then approximated in $W^{1,2}(Z)$ by the antisymmetric extended functions $h \in \operatorname{Lip}(Z)$, and so $f \in W^{1,2}(Z)$. 
Because $\operatorname{tr}_{Z_{0}} u=\operatorname{tr}_{Z_{0}} f=0$, the antisymmetric extension $u$ is of class $W^{1,2}(Z)$, like that of $f$. We show that $u$ is weakly harmonic in the interior $U$ of $Z$. For any antisymmetric function $\lambda \in \operatorname{Lip}_{c}(U)$ we have $\operatorname{tr}_{Z_{0}} \lambda=0$, and hence $\lambda_{\mid U_{+}} \in$ $W_{0}^{1,2}\left(U_{+}\right)$by Lemma 1 (b), $U_{+}=Z_{+} \backslash Z_{0}$ denoting the interior of $Z_{+}$relative to $Z$. Because $u$ is weakly harmonic in the open set $U_{+}(\subset X \backslash b X)$ it follows that $\int_{U_{+}}\langle\nabla u, \nabla \lambda\rangle d \mu=0$, and similarly with $U_{+}$replaced by $U_{-}:=\tau\left(U_{+}\right)$, or even by $U=U_{+} \cup U_{-} \cup\left(U \cap Z_{0}\right)$ because $\mu\left(Z_{0}\right)=0$. For a symmetric function $\lambda \in \operatorname{Lip}_{c}(U)$, $\langle\nabla u, \nabla \lambda\rangle$ is antisymmetric, and so we have again $\int_{U}\langle\nabla u, \nabla \lambda\rangle d \mu=0$. We conclude that $u$ is indeed weakly harmonic in $U$, and therefore has a continuous (harmonic) version in $U$ [EF, Theorem 6.2], necessarily equal to $f=0$ on $U \cap Z_{0}$. Consequently, (4.1) holds in the present case.

Step 2. This is the general case, where it is merely required that $f_{\mid b X}$ be continuous at the given point $x_{0}$, and that $f\left(x_{0}\right)=0$. For $\epsilon>0$ consider, as in $\mathrm{EF}$. $\mathrm{p}$. $67]$, the odd function $T_{\epsilon}: \mathbb{R} \rightarrow \mathbb{R}$ supported by $[-2 \epsilon, 2 \epsilon]$, and defined for $s \geq 0$ by

$$
T_{\epsilon}(s)= \begin{cases}s & \text { for } 0 \leq s \leq \epsilon \\ 2 \epsilon-s & \text { for } \epsilon \leq s \leq 2 \epsilon\end{cases}
$$

Each $T_{\epsilon}$ is a normal contraction of $\mathbb{R}$, operating on $W^{1,2}(X)$ EF Definition 2.5 and Proposition 5.1]. For any $f \in W^{1,2}(X)$ the function $f_{j}:=f-T_{1 / j} \circ f(j=1,2, \ldots)$ is thus of class $W^{1,2}(X)$, and $f_{j}=0$ in a neighbourhood $V_{j}$ of $x_{0}$ relative to $b X$ because $f(x) \rightarrow 0$ as $x \rightarrow x_{0}$ in $b X$. By Step 1 the unique harmonic function $u_{j}$ on $X \backslash b X$ such that $\left(u_{j}-f_{j}\right)_{\mid X \backslash b X} \in W_{0}^{1,2}(X \backslash b X)$ therefore satisfies $u_{j}(x) \rightarrow 0$ as $x \rightarrow x_{0}, x \in X \backslash b X$. From $\left|f_{j}-f\right| \leq 1 / j$ follows $\left|u_{j}-u\right| \leq 1 / j$ in $X \backslash b X$ by the last but one assertion of [EF, Theorem 5.2] (applied with $X$ replaced by $X \backslash b X$ ). Thus $u_{j} \rightarrow u$ uniformly in $X \backslash b X$ as $j \rightarrow \infty$, and (4.1) follows.

If, in addition, $f$ is subharmonic in $X \backslash b X$, it follows from the very last assertion of [EF, Theorem 5.2] that $f \leq u$ in $X \backslash b X$, and hence by (4.1)

$$
\limsup _{X \ni x \rightarrow x_{0}} f(x) \leq 0
$$

noting that $f(x) \rightarrow 0$ as $x \rightarrow x_{0}$ in $b X$. Under the hypotheses on $Y$ and $\phi$ in the theorem this applies to the distance function $f(x)=d_{Y}\left(\phi(x), \phi\left(x_{0}\right)\right)$, which is of class $W^{1,2}(X)$ by [EF, Corollaries 9.1, 9.2]; (Hölder) continuous in $X \backslash b X$, like $\phi$, and continuous at $x_{0}$ relative to $b X$ with the value 0 , by hypothesis on $\phi$. According to [EF, Lemma 10.2 (a)] (likewise applied with $X$ replaced by $X \backslash b X$ ) $f$ is weakly subharmonic in $X \backslash b X$, and indeed subharmonic there, by continuity follows from (4.2) that $d_{Y}\left(\phi(x), \phi\left(x_{0}\right)\right) \rightarrow 0$, i.e., $\phi(x) \rightarrow \phi\left(x_{0}\right)$ as $x \rightarrow x_{0}$ in $X$.

Proof of (b). Replacing $X \backslash b X$ by any admissible Riemannian polyhedron $(X, g)$, we show more generally that $d_{Y}\left(\phi_{0}, \phi_{1}\right)$ is subharmonic in $X$ for any two harmonic maps $\phi_{0}, \phi_{1}:(X, g) \rightarrow\left(Y, d_{Y}\right)$, or equivalently: two continuous, locally E-minimizing maps EF, Lemma 12.1].

Since $\left(Y, d_{Y}\right)$ is a simply connected complete geodesic space of nonpositive curvature, so is $\left(Y \times Y, d_{Y \times Y}\right)$ with the "Pythagorean" metric defined by

$$
d_{Y \times Y}^{2}\left(\left(y_{0}, y_{1}\right),\left(z_{0}, z_{1}\right)\right)=d_{Y}^{2}\left(y_{0}, z_{0}\right)+d_{Y}^{2}\left(y_{1}, z_{1}\right)
$$

\footnotetext{
${ }^{7}$ The fact that every continuous weakly subharmonic function $f$ is subharmonic follows, e.g., from [EF] Proposition 5.3 (ii)], applied to $u=f-H_{f}^{V}$ in any regular domain $V$. See also [EF, Remark 7.4].
} 
EF Lemma 12.6]. (Recall that a complete geodesic space $\left(Y, d_{Y}\right)$ is simply connected with nonpositive curvature if and only if $\left(Y, d_{Y}\right)$ has globally nonpositive curvature; cf. e.g. [EF, Chapter 2].)

A map $\phi_{0} \times \phi_{1}: X \rightarrow Y \times Y$, that is, $x \mapsto\left(\phi_{0}(x), \phi_{1}(x)\right)$, has according to Definition 1 (with the supremum over $f \in C_{c}(X,[0,1])$ viewed as a limit) the energy

$$
E\left(\phi_{0} \times \phi_{1}\right)=E\left(\phi_{0}\right)+E\left(\phi_{1}\right) .
$$

It follows that $\phi_{0} \times \phi_{1}$ is locally $E$-minimizing if $\phi_{0}$ and $\phi_{1}$ are also.

The permutation $\left(y_{0}, y_{1}\right) \mapsto\left(y_{1}, y_{0}\right)$ is an isometric involution of $Y \times Y$, and

$$
d_{Y \times Y}^{2}\left(\phi_{0} \times \phi_{1}, \phi_{1} \times \phi_{0}\right)=2 d_{Y}^{2}\left(\phi_{0}, \phi_{1}\right) .
$$

The claim in Part (b) therefore amounts to $d_{Y \times Y}\left(\phi_{0} \times \phi_{1}, \phi_{1} \times \phi_{0}\right)$ being subharmonic. In other words, we have reduced Part (b) to the particular case where $Y$ admits an isometric involution $y \mapsto \check{y}$, and where $\phi_{1}=\left(\phi_{0}\right)^{\sim}$ (i.e., $\phi_{1}(x)=\left(\phi_{0}(x)\right)^{\sim}$, $x \in X)$.

For $x, x^{\prime} \in X$ consider the following (ordered) quadrilateral

$$
P Q R S=\phi_{0}(x) \phi_{0}\left(x^{\prime}\right) \phi_{1}\left(x^{\prime}\right) \phi_{1}(x)
$$

in $Y$. Then $S=\check{P}, R=\check{Q}$, and so $P Q=R S$ and $P R=Q S$. By Reshetnyak's majorization theorem $\left[\mathrm{R}\right.$. (cf. [KS, Theorems 2.1.1 and 2.1.2]), there exists in $\mathbb{R}^{2}$ a convex comparison quadrilateral $\widetilde{P} \widetilde{Q} \widetilde{R} \widetilde{S}$ with the same side lengths and no shorter diagonals. In brief notation:

$$
\begin{gathered}
P Q=\widetilde{P} \widetilde{Q}=R S=\widetilde{R} \widetilde{S}, Q R=\widetilde{Q} \widetilde{R}, S P=\widetilde{S} \widetilde{P}, \\
P R \leq \widetilde{P} \widetilde{R}, Q S \leq \widetilde{Q} \widetilde{S},
\end{gathered}
$$

and more generally,

$$
P_{\lambda} Q_{\lambda^{\prime}} \leq \widetilde{P}_{\lambda} \widetilde{Q}_{\lambda^{\prime}}
$$

for $\lambda, \lambda^{\prime} \in[0,1]$, where $P_{\lambda}$ and $Q_{\lambda^{\prime}}$ are defined in $Y$ by

$$
P_{\lambda}=(1-\lambda) P+\lambda S, \quad Q_{\lambda^{\prime}}=\left(1-\lambda^{\prime}\right) Q+\lambda^{\prime} R,
$$

with the usual abuse of notation; and $\widetilde{P}_{\lambda}$ and $\widetilde{Q}_{\lambda^{\prime}}$ are defined similarly in $\mathbb{R}^{2}$. We show that it can be arranged that $\widetilde{P} \widetilde{R}=\widetilde{Q} \widetilde{S}$. By continuity, in proving this we need not consider degenerate cases (coalescence or collinearity among points). Among all convex quadrilaterals $\widetilde{P} \widetilde{Q} \widetilde{R} \widetilde{S}$ in $\mathbb{R}^{2}$ satisfying (4.4), there exists by compactness one in which $|\widetilde{P} \widetilde{R}-\widetilde{Q} \widetilde{S}|$ is minimal. If the minimum value is 0 we are done, for then $\widetilde{P} \widetilde{R}=\widetilde{Q} \widetilde{S}$. Suppose that, e.g., $\widetilde{P} \widetilde{R}>\widetilde{Q} \widetilde{S}$. Fixing $\widetilde{P}$ and $\widetilde{S}$, turn $\widetilde{P} \widetilde{Q}$ about $\widetilde{P}$ so as to slightly increase the angle of the quadrilateral $\widetilde{P} \widetilde{Q} \widetilde{R} \widetilde{S}$ at $\widetilde{P}$. Then $\widetilde{Q} \widetilde{S}$ increases (by the cosine relation for the triangle $\widetilde{Q} \widetilde{P} \widetilde{S}$ ), and so the angle at $\widetilde{R}$ increases as well (by the cosine relation for the triangle $\widetilde{Q} \widetilde{R} \widetilde{S}$ ). One (in fact both) of the two remaining angles at $\widetilde{Q}$ or $\widetilde{S}$ must therefore decrease, and hence $\widetilde{P} \widetilde{R}$ decreases. In this way $\widetilde{P} \widetilde{R}$ remains $>\widetilde{Q} \widetilde{S} \geq Q S=P R$, but $\widetilde{P} \widetilde{R}-\widetilde{Q} \widetilde{S}$ decreases, in contradiction with the minimality. Thus there does exist a convex quadrilateral $\widetilde{P} \widetilde{Q} \widetilde{R}$ in $\mathbb{R}^{2}$ satisfying (4.4) and $\widetilde{P} \widetilde{R}=\widetilde{Q} \widetilde{S}$. Being convex, $\widetilde{P} \widetilde{Q} \widetilde{R} \widetilde{S}$ is therefore symmetric about the line in $\mathbb{R}^{2}$ through the midpoints of $\widetilde{P} \widetilde{S}$ and $\widetilde{Q} \widetilde{R}$ (say the line $\mathbb{R} \times\{0\}$ ).

With $U$ as in the definition of a local $E$-minimizer (Section 1) (cf. [EF, Lemma 12.1]), consider a Lipschitz function $\lambda, 0 \leq \lambda \leq 1$, of compact support in $U$, and 
define

$$
\phi_{\lambda}(x)=(1-\lambda(x)) \phi_{0}(x)+\lambda(x) \phi_{1}(x), \quad x \in U .
$$

For brevity write

$$
d_{Y}\left(\phi_{0}(x), \phi_{1}(x)\right)=u(x)
$$

a function of class $W_{\text {loc }}^{1,2}(X)$ according to EF, Corollaries 9.1, 9.2] applied to the Lipschitz function $d_{Y}: Y \times Y \rightarrow \mathbb{R}$ and the map $\phi_{0} \times \phi_{1} \in W_{\text {loc }}^{1,2}(X, Y \times Y)$. Using (4.4) through (4.7) and taking $\lambda=\lambda(x), \lambda^{\prime}=\lambda\left(x^{\prime}\right)$ in (4.5) we obtain

$$
\begin{gathered}
d_{Y}^{2}\left(\phi_{\lambda}(x), \phi_{\lambda^{\prime}}\left(x^{\prime}\right)\right)-d_{Y}^{2}\left(\phi_{0}(x), \phi_{0}\left(x^{\prime}\right)\right)=P_{\lambda} Q_{\lambda^{\prime}}^{2}-P Q^{2} \leq \widetilde{P}_{\lambda} \widetilde{Q}_{\lambda^{\prime}}^{2}-\widetilde{P} \widetilde{Q}^{2} \\
=-\left[\lambda(x) u(x)-\lambda\left(x^{\prime}\right) u\left(x^{\prime}\right)\right]\left[(1-\lambda(x)) u(x)-\left(1-\lambda\left(x^{\prime}\right)\right) u\left(x^{\prime}\right)\right],
\end{gathered}
$$

where of course $\phi_{\lambda^{\prime}}\left(x^{\prime}\right):=\left(1-\lambda\left(x^{\prime}\right)\right) \phi_{0}\left(x^{\prime}\right)+\lambda\left(x^{\prime}\right) \phi_{1}\left(x^{\prime}\right)$. For the approximate energy densities $e_{\epsilon}\left(\phi_{0}\right)$ and $e_{\epsilon}\left(\phi_{\lambda}\right)$ (cf. (1.1)) we thus have

$$
\begin{aligned}
& e_{\epsilon}\left(\phi_{\lambda}\right)(x)-e_{\epsilon}\left(\phi_{0}\right)(x) \leq \\
& \frac{1}{\epsilon^{m+2}} \int_{B_{U}(x, \epsilon)}\left[-\lambda(x) u(x)+\lambda\left(x^{\prime}\right) u\left(x^{\prime}\right)\right]\left[(1-\lambda(x)) u(x)-\left(1-\lambda\left(x^{\prime}\right)\right) u\left(x^{\prime}\right)\right] d \mu\left(x^{\prime}\right) .
\end{aligned}
$$

This leads for $f \in C_{c}(U,[0,1])$ to

$$
\limsup _{\epsilon \rightarrow 0} \int_{U}\left(e_{\epsilon}\left(\phi_{\lambda}\right)-e_{\epsilon}\left(\phi_{0}\right)\right) f d \mu \leq c_{m} \int_{U}\langle\nabla(-\lambda u), \nabla(u-\lambda u)\rangle f d \mu
$$

according to (1.5) applied to $-\lambda u$ and $u-\lambda u$, both of class $W^{1,2}(U)$ EF, Remark 5.1 (a)]. Note that $\phi_{\lambda}=\phi_{0}$ off $F:=\operatorname{supp} \lambda$. By (1.2) applied to $\phi_{\lambda}$ and (1.3) applied to $\phi_{0}$ it follows from (1.6) and (4.8) (after restricting $\phi_{0}$ to $U$ ) that

$$
\begin{aligned}
0 & \leq E\left(\phi_{\lambda}\right)-E\left(\phi_{0}\right)=\int_{F}\left(e\left(\phi_{\lambda}\right)-e\left(\phi_{0}\right)\right) d \mu=E\left(\left(\phi_{\lambda}\right)_{\mid U}\right)-E\left(\left(\phi_{0}\right)_{\mid U}\right) \\
& =\lim _{f \in C_{c}(U,[0,1])} \limsup _{\epsilon \rightarrow 0} \int_{U}\left(e_{\epsilon}\left(\phi_{\lambda}\right)-e_{\epsilon}\left(\phi_{0}\right)\right) f d \mu \\
& \leq c_{m} \int_{U}\langle\nabla(-\lambda u), \nabla(u-\lambda u)\rangle d \mu .
\end{aligned}
$$

In the resulting inequality, replace $t$ by $\lambda t$ for small $t>0$, divide by $t$, and let $t \rightarrow 0$ :

$$
\int_{U}\langle\nabla(\lambda u), \nabla u\rangle d \mu \leq 0, \quad \lambda \in \operatorname{Lip}_{c}^{+}(U) .
$$

This should be compared with [EF, eq. (10.6)] (where $v=d_{Y}(\phi, q)$ corresponds to the present $\left.u=d_{Y}\left(\phi_{0}, \phi_{1}\right)\right)$. Thus it follows that $u$ is weakly subharmonic, and indeed subharmonic because $\phi_{0}, \phi_{1}$, and hence $u=d_{Y}\left(\phi_{0}, \phi_{1}\right)$ are continuous, see footnote 7 above. That simplifies the proof at this point: the set $U_{n}$ on [EF, p. 184], becomes a usual open set, and so there is no need for fine potential theory here. (Similarly concerning the proof of Theorem 3 in Section 8.)

\section{Proof of Theorem 2}

For $y \in Y$ let $p_{C}(y)=p(y)$ denote the unique point of $C$ nearest to $y$ (cf. [KS. Proposition 2.5.4]); thus $d_{Y}(y, C)=d_{Y}(y, p(y))$. 
Proof of (a). For $y, y^{\prime} \in Y \backslash C$ consider the (ordered) quadrilateral $y, y^{\prime}, p\left(y^{\prime}\right), p(y)$ in $Y$. As shown by Reshetnyak [ $[\mathrm{R}]$ (cf. [KS, Theorem 2.1.1]), there exists in $\mathbb{R}^{2}$ a convex quadrilateral $P Q R S$ with the same side lengths and no shorter diagonals. For $\lambda, \lambda^{\prime} \in[0,1]$ write (with a standard abuse of notation)

$$
y_{\lambda}=(1-\lambda) y+\lambda p(y), \quad y_{\lambda^{\prime}}^{\prime}=\left(1-\lambda^{\prime}\right) y^{\prime}+\lambda^{\prime} p\left(y^{\prime}\right),
$$

and similarly in $\mathbb{R}^{2}: P_{\lambda}=(1-\lambda) P+\lambda S, Q_{\lambda^{\prime}}=\left(1-\lambda^{\prime}\right) Q+\lambda^{\prime} R$. Then

$$
P_{\lambda} Q_{\lambda^{\prime}} \geq d_{Y}\left(y_{\lambda}, y_{\lambda^{\prime}}^{\prime}\right)
$$

again according to $[\underline{\mathrm{R}}$; cf. $[\mathrm{KS}$, Theorem 2.1.2].

We show that (if $R \neq S$ ) the angles $\eta$ and $\eta^{\prime}$ in $P Q R S$ at $S$ and $R$, respectively, are both $\geq \pi / 2$. Taking $\lambda^{\prime}=1$ and hence $y_{\lambda^{\prime}}^{\prime}=p\left(y^{\prime}\right), Q_{\lambda^{\prime}}=R$ in (5.1) we obtain

$$
P_{\lambda} R=P_{\lambda} Q_{1} \geq d_{Y}\left(y_{\lambda}, p\left(y^{\prime}\right)\right) \geq d_{Y}\left(p(y), p\left(y^{\prime}\right)\right)=R S,
$$

the latter inequality because $p\left(y_{\lambda}\right)=p(y)$ and because $p$ is contractive (and idempotent) [KS, Proposition 2.5.4]. For $\lambda \rightarrow 1$ this shows, e.g. by the cosine relation, that indeed $\eta \geq \pi / 2$. Taking instead $\lambda=1$ leads similarly for $\lambda^{\prime} \rightarrow 1$ to $\eta^{\prime} \geq \pi / 2$.

From $P Q R S$ we construct a further convex quadrilateral $\widetilde{P} \widetilde{Q} \widetilde{R} \widetilde{S}$ in $\mathbb{R}^{2}$, now with the angles at $\widetilde{S}$ and $\widetilde{R}$ equal to $\pi / 2$, and with three side lengths preserved:

$$
\widetilde{P} \widetilde{Q}=P Q, \widetilde{P} \widetilde{S}=P S, \widetilde{Q} \widetilde{R}=Q R .
$$

Taking $\widetilde{P}=P$ and $\widetilde{Q}=Q$ we consider the circles in $\mathbb{R}^{2}$ centered at $P$, resp. $Q$, and passing through $S$, resp. $R$. Then $\widetilde{S}$ and $\widetilde{R}$ are uniquely determined (up to reflexion in the line through $P$ and $Q$ ) as the points of contact between these two circles and one of their common tangents not separating $P$ and $Q$. None of these two circles encloses the other because $P S=d_{Y}(y, p(y)) \leq d_{Y}\left(y, p\left(y^{\prime}\right)\right) \leq P R \leq P Q+Q R$, and similarly $Q R \leq P Q+P S$.

Writing $\widetilde{P}_{\lambda}=(1-\lambda) \widetilde{P}+\lambda \widetilde{S}$ and $\widetilde{Q}_{\lambda^{\prime}}=\left(1-\lambda^{\prime}\right) \widetilde{Q}+\lambda^{\prime} \widetilde{R}$, one easily finds from $\eta, \eta^{\prime} \geq \pi / 2$ that

$$
\widetilde{P}_{\lambda} \widetilde{Q}_{\lambda^{\prime}} \geq P_{\lambda} Q_{\lambda^{\prime}}
$$

(This also holds if the quadrilateral $P Q R S$ degenerates.)

With $U$ as in the definition of a local $E$-minimizer (Section 1) (cf. EF, Lemma 12.1]), consider a function $\lambda \in \operatorname{Lip}_{c}(U), 0 \leq \lambda \leq 1$, and apply the above to

$$
\lambda=\lambda(x), \lambda^{\prime}=\lambda\left(x^{\prime}\right), y=\phi(x), y^{\prime}=\phi\left(x^{\prime}\right), y_{\lambda}=\phi_{\lambda}(x), y_{\lambda^{\prime}}^{\prime}=\phi_{\lambda^{\prime}}\left(x^{\prime}\right)
$$

for $x \in U, x^{\prime} \in B_{U}(x, \epsilon), \epsilon>0$; here we set $\phi_{\lambda}(x)=(1-\lambda(x)) \phi(x)+\lambda(x) p(\phi(x))$ and similarly $\phi_{\lambda^{\prime}}\left(x^{\prime}\right)=\left(1-\lambda\left(x^{\prime}\right)\right) \phi\left(x^{\prime}\right)+\lambda\left(x^{\prime}\right) p\left(\phi\left(x^{\prime}\right)\right)$. Also write

$$
\begin{gathered}
d=d_{Y}\left(\phi(x), \phi\left(x^{\prime}\right)\right)=\widetilde{P} \widetilde{Q}, \quad d_{\lambda}=d_{Y}\left(\phi_{\lambda}(x), \phi_{\lambda^{\prime}}\left(x^{\prime}\right)\right) \leq \widetilde{P}_{\lambda} \widetilde{Q}_{\lambda^{\prime}}, \\
u=u(x)=d_{Y}(\phi(x), C)=\widetilde{P} \widetilde{S}, \quad u^{\prime}=u\left(x^{\prime}\right)=d_{Y}\left(\phi\left(x^{\prime}\right), C\right)=\widetilde{Q} \widetilde{R},
\end{gathered}
$$

where we have used (5.1), (5.2), (5.3) for the inequality in (5.4). For the convex quadrilateral $\widetilde{P} \widetilde{Q} \widetilde{R} \widetilde{S}$ in $\mathbb{R}^{2}$ we then have from (5.3), (5.4), (5.5)

$$
d_{\lambda}^{2}-d^{2} \leq \widetilde{P}_{\lambda} \widetilde{Q}_{\lambda^{\prime}}^{2}-\widetilde{P} \widetilde{Q}^{2}=\left[(1-\lambda) u-\left(1-\lambda^{\prime}\right) u^{\prime}\right]^{2}-\left(u-u^{\prime}\right)^{2} .
$$


For the approximate energy densities $e_{\epsilon}(\phi)$ and $e_{\epsilon}\left(\phi_{\lambda}\right)$ (cf. (1.1)), we therefore obtain

$$
\begin{aligned}
& e_{\epsilon}\left(\phi_{\lambda}\right)(x)-e_{\epsilon}(\phi)(x) \\
& \quad \leq \frac{1}{\epsilon^{m+2}} \int_{B_{U}(x, \epsilon)}\left(\left[(1-\lambda(x)) u(x)-\left(1-\lambda\left(x^{\prime}\right)\right) u\left(x^{\prime}\right)\right]^{2}-\left[u(x)-u\left(x^{\prime}\right)\right]^{2}\right) d \mu\left(x^{\prime}\right) .
\end{aligned}
$$

By integration over $U$ we get for $\epsilon \rightarrow 0$ from (1.2), (1.3), (1.4) (applied with $U$ in place of $X$ after restricting $\phi$ to $U$; cf. (1.6))

$$
\begin{aligned}
0 \leq E\left(\phi_{\lambda}\right)-E(\phi) & \leq c_{m} \int_{U}\left(|\nabla(u-\lambda u)|^{2}-|\nabla u|^{2}\right) d \mu \\
& =c_{m} \int_{U}\left(-2\langle\nabla u, \nabla(\lambda u)\rangle+|\nabla(\lambda u)|^{2}\right) d \mu .
\end{aligned}
$$

For details about this, compare with the analogous deduction of (4.9) via (4.8).

In the resulting inequality replace $\lambda$ by $t \lambda$ for small $t>0$, divide by $t$, and let $t \rightarrow 0$ :

$$
\int_{U}\langle\nabla u, \nabla(\lambda u)\rangle d \mu \leq 0, \quad \lambda \in \operatorname{Lip}_{c}^{+}(U) .
$$

This should be compared with $\left[\mathrm{EF}\right.$, eq. (10.6)] (where $v=d_{Y}(\phi, q)$ corresponds to the present $\left.u=d_{Y}(\phi, C)\right)$. In this way we conclude that $u$ is weakly subharmonic, and indeed subharmonic, by continuity (cf. footnote 7 in Section 4 ).

Proof of (b). Like $Y$, the product $Y \times \mathbb{R}$ is a simply connected complete geodesic space of nonpositive curvature, with the "Pythagorean" metric given by

$$
d_{Y \times \mathbb{R}}^{2}\left(\left(y_{0}, t_{0}\right),\left(y_{1}, t_{1}\right)\right)=d_{Y}^{2}\left(y_{0}, y_{1}\right)+\left|t_{0}-t_{1}\right|^{2}
$$

EF Lemma 12.6]. For any relatively compact open set $U \subset X$ the map $\phi_{\mid U} \times 0$ : $U \rightarrow Y \times \mathbb{R}$ given by $x \mapsto(\phi(x), 0)$ has clearly the same energy as $\phi_{\mid U}$. Since $\phi$ is locally $E$-minimizing, so therefore is $\phi \times 0$; cf. the third paragraph of the subsection Proof of (b), in Section 4. By Part (a) the distance function $u=d_{Y \times \mathbb{R}}(\phi \times 0, C)$ is subharmonic for any closed convex set $C \subset Y \times \mathbb{R}$.

Given a continuous convex function $f>0$ on an open set $V \subset Y$, and a point $x_{0} \in \phi^{-1}(V)$, consider the closed convex epigraph $C_{n}$ of the restriction of $f / n$, $n=1,2, \ldots$, to a compact neighbourhood $Q$ of $\phi\left(x_{0}\right)$ in $V$ :

$$
C_{n}=\{(y, t) \in Q \times \mathbb{R}: t \geq f(y) / n\},
$$

and the subharmonic function $u_{n}:=n d_{Y \times \mathbb{R}}\left(\phi \times 0, C_{n}\right)$ on $U:=\phi^{-1}\left(Q^{\circ}\right)$ (an open neighbourhood of $x_{0}$ ); thus

$$
u_{n}^{2}(x)=\min _{z \in Q}\left\{n^{2} d_{Y}^{2}(\phi(x), z)+f^{2}(z)\right\} \leq(f \circ \phi)^{2}(x), \quad x \in U
$$

(take $z=\phi(x)$ ). Denote $z_{n}=z_{n}(x)$ the point $z$ of $Q$ realizing the minimum (5.6), i.e., $\left(z_{n}, f\left(z_{n}\right) / n\right)$ is the point of $C_{n}$ nearest to $(\phi(x), 0)$.

It follows from (5.6) that the sequence $\left(u_{n}(x)\right)$ is increasing. Furthermore,

$$
\max \left\{n d_{Y}\left(\phi(x), z_{n}\right), f\left(z_{n}\right)\right\} \leq u_{n}(x) \leq(f \circ \phi)(x), \quad x \in U .
$$

Consequently, $z_{n} \rightarrow \phi(x)$ as $n \rightarrow \infty$, and hence $f\left(z_{n}\right) \rightarrow(f \circ \phi)(x)$. We conclude that $u_{n}(x) \nearrow(f \circ \phi)(x)$ as $n \rightarrow \infty$, and since $f \circ \phi$ is continuous and the $u_{n}$ are subharmonic, $f \circ \phi$ is likewise subharmonic in $U$, and hence in all of $\phi^{-1}(V)$. 


\section{Proof of Proposition 1}

In view of Theorem 2 (b), only the "if part" remains. Let $U$ denote the (open) star of a vertex of $X$ in a triangulation sufficiently fine that $U$ is the interior of its closure (cf. [EF, Remark 4.2]); then $\partial U=b U$. Let $\psi$ be the continuous variational solution to the Dirichlet problem in $\bar{U}$ with continuous boundary map $\psi_{\mid \partial U}=\phi_{\mid \partial U}$; cf. [EF, Theorem 11.3] and the present Theorem 1. Since the stars $U$ cover $X$, it suffices to prove that $\psi=\phi$ in $U$, i.e., $d_{Y}(\phi, \psi) \equiv 0$ in $U$, for then $\phi$ is harmonic in each $U$ and hence in all of $X$. And for that we show that $d_{Y}(\phi, \psi)$ is subharmonic at each point $x_{0}$ of $U$; cf. Theorem 1 (b). We may assume that $\phi\left(x_{0}\right) \neq \psi\left(x_{0}\right)$. Let $\gamma:[0,1] \rightarrow Y$ be the constant-speed geodesic in $Y$ with endpoints $\gamma(0)=\phi\left(x_{0}\right)$, $\gamma(1)=\psi\left(x_{0}\right)$.

Suppose first that $\operatorname{dim} Y=2$ and, for some $0<\tau<1, \gamma(\tau)$ belongs to an open 2 -simplex $s$ of the target polyhedron $(Y, h)$. Let $B$ denote a Riemannian ball in $Y$ (necessarily convex), centered at $\gamma(\tau)$ and contained in $s$; and let $C$ be the geodesic segment in $B$ through $\gamma(\tau)$ and perpendicular to $\gamma$ at $\gamma(\tau)$ for the Riemannian metric $h_{\mid B}$ on $B$, and hence for the intrinsic distance $d_{Y}$ on $Y$, in particular on $B$ [Jo1, Definition 2.1 and Lemma 2.2]. Then $\gamma$ is perpendicular to $C$ at $\gamma(\tau)$ [Jo1. Lemma 2.3]. Since $C$ is convex, it follows that $p_{C}\left(\phi\left(x_{0}\right)\right)=\gamma(\tau)$. For $x \in U$ the point $p_{C}(\phi(x))=p(\phi(x))$ of $C$ nearest to $\phi(x)$ therefore converges to $\gamma(\tau)$ as $x \rightarrow x_{0}$ because $\phi$ and $p$ are continuous [KS, Proposition 2.5.4]. Similarly as to $p(\psi(x))$.

Moreover, the geodesic segment $\phi(x) \psi(x)$ converges to $\phi\left(x_{0}\right) \psi\left(x_{0}\right)$ as $x \rightarrow x_{0}$, and has therefore some point $\kappa(x)$ in common with $C$ for $x$ near $x_{0}$. Then

$$
\begin{aligned}
d_{Y}(\phi(x), \psi(x)) & =d_{Y}(\phi(x), \kappa(x))+d_{Y}(\kappa(x), \psi(x)) \\
& \geq d_{Y}(\phi(x), C)+d_{Y}(\psi(x), C) .
\end{aligned}
$$

But $d_{Y}(\phi, C)$ and $d_{Y}(\psi, C)$ are subharmonic in $U$, the latter by Theorem 2 (a) since $\psi$ is harmonic in $U$, and the former by hypothesis since $d_{Y}(\cdot, C)$ is convex, $\left(Y, d_{Y}\right)$ being globally squared-convex; cf. e.g. [EF, p. 26]. For $x=x_{0}$ there is equality in (6.1) because $p\left(\phi\left(x_{0}\right)\right)=\gamma(\tau)=p\left(\psi\left(x_{0}\right)\right)$, as noted above. It follows that the "meanvalue" inequality in the definition of a subharmonic function (cf. e.g. [EF, Definition 2.2]), holds at $x_{0}$ for the function $d_{Y}(\phi, \psi)$ because it holds for the subharmonic minorant $d_{Y}(\phi, C)+d_{Y}(\psi, C)$ in $U$ with the same value at $x_{0}$.

In the remaining case there exists $0<\tau<1$ and an open interval $J$ containing $\tau$ such that $\gamma(J)$ is contained in some open 1-simplex $\sigma$ of the polyhedron $Y$, with adjacent open 2-simplexes $s_{1}, \ldots, s_{k}$ of $Y$ in case $\operatorname{dim} Y=2$. Let $B$ denote a closed ball centered at $\gamma(\tau)$ and contained in the star of $\gamma(\tau)$ in $Y$. This time let $C$ be the union of the geodesics $C_{i} \subset B \cap\left(s_{i} \cup \sigma\right)$ issuing from $\gamma(\tau), i=1, \ldots, k$, and perpendicular to $\gamma(J)$ in $s_{i} \cup \sigma$. These geodesics $C_{i}$ exist because $h$ is simplexwise smooth; cf. the paragraph containing (1.1). Again, $C$ is convex, now because $C_{i} \cup C_{j}$ is a geodesic in $Y$ for any two $i \neq j$. (Indeed, for points $y_{i} \in C_{i} \backslash\{\gamma(\tau)\}$ and $y_{j} \in C_{j} \backslash\{\gamma(\tau)\}$ with sufficiently small distances $\epsilon_{i}$ and $\epsilon_{j}$ from $\gamma(\tau)$, the balls $B_{Y}\left(y_{i}, \epsilon_{i}\right)$ and $B_{Y}\left(y_{j}, \epsilon_{j}\right)$ are contained in $s_{i} \cup\{\gamma(\tau)\}$ and $s_{j} \cup\{\gamma(\tau)\}$, respectively. For $y \in \gamma(J) \backslash\{\gamma(\tau)\}$ it follows that $d_{Y}\left(y_{i}, y\right)+d_{Y}\left(y_{j}, y\right)>\epsilon_{i}+\epsilon_{j} \geq d_{Y}\left(y_{i}, y_{j}\right)$.) Again, $\gamma$ is perpendicular to $C_{i} \cup C_{j}$ at $\gamma(\tau)$, by [Jo1, Lemma 2.3], and hence to $C$ (in the sense that $\left.p_{C}\left(\phi\left(x_{0}\right)\right)=\gamma(\tau)\right)$. The previous argument therefore carries over. (If $\operatorname{dim} Y=1, C$ reduces to the point $\gamma(\tau)$.) 
Attempting to extend this proof to higher dimensional polyhedral (or geodesic) targets $Y$ would require replacing $C$ by the union of all geodesics in $Y$ perpendicular to $\gamma$ at $\gamma(\tau)$, but that union generally fails to be convex (even near $\gamma(\tau)$ ).

\section{Proof of Lemma 2}

Proof of (a). Consider a quadrilateral $P Q R S$ in $B$ satisfying $P Q, R S \leq \pi / 2$, as well as (2.5): $2(P Q \diamond R S)+Q R+P S<2 \pi$; cf. (2.3). We shall construct in $S^{2}$ a comparison trapezoid $\widetilde{P} \widetilde{Q} \widetilde{R} \widetilde{S}$ for $P Q R S$; cf. (2.4). For brevity write

$$
P Q=a, R S=b, P S=c, Q R=d, P R=h, Q S=k .
$$

From (2.5) follows $a+b+c+d<2 \pi$ because $\frac{1}{2}(a+b) \leq a \diamond b$. Clearly, $a, b, c, d, h, k<\pi$ since the diameter of $B$ is $\leq 2 R<\pi$. In view of Reshetnyak's majorization theorem $\left[\mathrm{R}\right.$ we may therefore suppose from the beginning that $Y=S^{2}$ and that $P Q R S$ is convex. The construction for that case will be done in four steps, in each of which $a \diamond b, c$, and $d$ will remain unaltered, while $h \diamond k$ will become larger (or remain the same).

Let $T$ denote the $3 \times 4$ matrix with columns $P, Q, R, S \in S^{2} \subset \mathbb{R}^{3}$, and $T^{*}$ its transpose; then the $4 \times 4$ matrix

$$
A=A(a, b, c, d, h, k):=\left(\begin{array}{cccc}
1 & \cos a & \cos h & \cos c \\
\cos a & 1 & \cos d & \cos k \\
\cos h & \cos d & 1 & \cos b \\
\cos c & \cos k & \cos b & 1
\end{array}\right)=T^{*} T
$$

is positive semidefinite of $\operatorname{rank} \operatorname{rk} A=\operatorname{rk} T \leq 3$ (hence $A$ is not positive definite).

Step 1. Interchanging $P, S$, and likewise $Q, R$, leads to a new convex quadrilateral $S R Q P$ in $S^{2}$ with associated matrix $A^{\#}$ obtained from $A$ by interchanging $a, b$ and likewise interchanging $h, k$, while keeping $c$ and $d$. Since $A$ and $A^{\#}$ are positive semidefinite, so is the matrix

$$
A_{1}:=\frac{1}{2}\left(A+A^{\#}\right)=A\left(a_{1}, b_{1}, c_{1}, d_{1}, h_{1}, k_{1}\right),
$$

where $a_{1}=b_{1}=a \diamond b, c_{1}=c, d_{1}=d, h_{1}=k_{1}=h \diamond k$; but $A_{1}$ may well have rank 4 and thus be positive definite.

Step 2. The positive semidefinite matrix $A_{1}$ with diagonal entries 1 can be embedded in $S^{3}$ (the unit sphere in $\mathbb{R}^{4}$ ); that is, there exists a quadrilateral $P_{1} Q_{1} R_{1} S_{1}$ in $S^{3}$ with side lengths $a_{1}, b_{1}, c_{1}, d_{1}$ and diagonals $h_{1}, k_{1}$. Here is the easy proof (cf. $[\mathrm{Sbg}]$ ): There exists a $4 \times 4$ matrix $T_{1}$ such that $T_{1}^{*} T_{1}=A_{1}$; the columns of $T_{1}$ serve as $P_{1}, Q_{1}, R_{1}, S_{1}$.

Step 3. In particular, $a_{1}+b_{1}+c_{1}+d_{1}=2(a \diamond b)+c+d<2 \pi$, by hypothesis. We show that $P_{1} Q_{1} R_{1} S_{1}$ is contained in a ball of radius $<\pi / 2$ in the Riemannian manifold $S^{3}$. Such a ball is of course geodesically convex and satisfies bipoint uniqueness. Thus $\left[\underline{\mathrm{R}}\right.$ applies again, now with $Y$ replaced by $S^{3}$ (having Alexandrov curvature $\leq 1$ ), and that produces a convex quadrilateral $P_{2} Q_{2} R_{2} S_{2}$ in $S^{2}$ with unchanged side lengths $a_{2}, b_{2}, c_{2}, d_{2}$ and with diagonals $h_{2} \geq h_{1}, k_{2} \geq k_{1}\left(=h_{1}\right)$.

To prove that such a ball in $S^{3}$ exists, note that for example $P_{1}$ and $Q_{1}$ are symmetric about a linear 3 -space $V\left(P_{1}, Q_{1}\right)$ in $\mathbb{R}^{4}$. Then $V\left(P_{1}, Q_{1}\right) \cap V\left(Q_{1}, R_{1}\right) \cap$ $V\left(R_{1}, S_{1}\right)$ is (or contains) a linear 1 -space in $\mathbb{R}^{4}$, meeting $S^{3}$ in two antipodal points $L, L^{\prime}$, say with $L P_{1} \leq \pi / 2$; then $L P_{1}=L Q_{1}=L R_{1}=L S_{1} \leq \pi / 2$. If $L P_{1}<\pi / 2$, we are done. If $L P_{1}=\pi / 2, P_{1} Q_{1} R_{1} S_{1}$ lies on the great 2 -sphere $S^{2}$ in $S^{3}$ with poles $L, L^{\prime}$. Working in this $S^{2}$, and invoking the relations $P_{1} Q_{1}=R_{1} S_{1}(=a \diamond b)$ 
and $P_{1} R_{1}=Q_{1} S_{1}(=h \diamond k)$, we see that the triangles $P_{1} Q_{1} S_{1}$ and $S_{1} R_{1} P_{1}$ are congruent (though possibly not with the same orientation).

If $P_{1} Q_{1} R_{1} S_{1}$ is nonconvex (in $S^{2}$ ), then $P_{1} S_{1}$ and $Q_{1} R_{1}$ have the same midpoint $M$, and $P_{1} Q_{1} R_{1} S_{1}$ is contained in the ball in $S^{2}$ centered at $M$ and with radius $\max \left\{M P_{1}, M Q_{1}\right\}<\pi / 2$, hence also contained in the corresponding ball in $S^{3} \supset S^{2}$.

In the remaining case, where $P_{1} Q_{1} R_{1} S_{1}$ is convex, $P_{1}, Q_{1}$ and $S_{1}, R_{1}$ are symmetric about a great circle $\gamma$ in $S^{2}$ (by the congruence of $P_{1} Q_{1} S_{1}$ and $S_{1} R_{1} P_{1}$ ). Furthermore, $P_{1}$ and $Q_{1}$ are symmetric about a great circle $\gamma^{\prime}$, and $\gamma \cap \gamma^{\prime}$ is (or contains) a pair of antipodal points $N, N^{\prime}$. If, for example, $N P_{1} \leq \pi / 2$, then $N P_{1}=N Q_{1}=N R_{1}=N S_{1} \leq \pi / 2$, and we are done in case $N P_{1}<\pi / 2$. Finally, if $N P_{1}=\pi / 2, P_{1} Q_{1} R_{1} S_{1}$ lies on a great circle $S^{1}$ in $S^{2}$; and we may assume that $P_{1} Q_{1}+Q_{1} R_{1}<\pi$ since $P_{1} Q_{1} R_{1} S_{1}$ is convex with perimeter $<2 \pi$. Then $Q_{1}$ lies on the arc $P_{1} R_{1}$ of length $<\pi$; and $P_{1} Q_{1}+Q_{1} R_{1}+R_{1} S_{1}<\pi$, for otherwise the perimeter of $P_{1} Q_{1} R_{1} S_{1}$ would be $2 \pi$. Now take as center the midpoint of $P_{1} S_{1}$ in $S^{1}\left(\subset S^{3}\right)$ and as radius $\frac{1}{2} P_{1} S_{1}\left(<\frac{1}{2} \pi\right)$.

Step 4 . Here we further achieve that the diagonals in the convex quadrilateral $P_{2} Q_{2} R_{2} S_{2}$ in $S^{2}$ from the first paragraph of Step 3 become equal. Suppose, for example, that $h_{2}>k_{2}$. We may then slightly reduce $h_{2}$ and enlarge $k_{2}$ while keeping $a_{2}\left(=b_{2}\right), c_{2}, d_{2}$ unaltered. Fixing $P_{2}$ and $S_{2}$, this is done by slightly increasing the angle at $P_{2}$, whereby the angle at $S_{2}$ decreases, as it is easily seen by invoking the convexity of the quadrilateral $P_{2} Q_{2} R_{2} S_{2}$. This can be continued until we obtain the desired convex quadrilateral $\widetilde{P} \widetilde{Q} \widetilde{R} \widetilde{S}$ in $S^{2}$ with side lengths $\tilde{a}=\tilde{b}=a \diamond b, \tilde{c}=c$, $\tilde{d}=d$, and diagonals $\tilde{h}=\tilde{k} \geq k_{2} \geq k_{1}=h \diamond k$.

Proof of (b). Again we may assume, by $\left[\mathrm{R}\right.$, that $Y=S^{2}$ and that $P Q R S$ is convex. We begin by determining $a^{*}:=P_{\lambda} Q$ from the spherical cosine relation for the triangles $Q P S$ and $Q P P_{\lambda}$ by eliminating the common angle at $P$, and similarly determining $b^{*}:=S_{\lambda} R$ from the triangles $R S P$ and $R S S_{\lambda}$ :

$$
\begin{aligned}
& \sin c \cos a^{*}=\sin (c-\lambda c) \cos a+\sin (\lambda c) \cos k, \\
& \sin c \cos b^{*}=\sin (c-\lambda c) \cos b+\sin (\lambda c) \cos h .
\end{aligned}
$$

In particular, we may determine

$$
h^{*}:=R P_{\lambda}=R S_{1-\lambda}
$$

by replacing $\lambda$ by $1-\lambda$ in (7.2); and similarly $k^{*}:=Q S_{\lambda}=Q P_{1-\lambda}$ from (7.1):

$$
\begin{aligned}
& \sin c \cos h^{*}=\sin (\lambda c) \cos b+\sin (c-\lambda c) \cos h, \\
& \sin c \cos k^{*}=\sin (\lambda c) \cos a+\sin (c-\lambda c) \cos k .
\end{aligned}
$$

If $\lambda \leq \frac{1}{2}$ the quadrilateral $Q P_{\lambda} S_{\lambda} R$ is convex, and so $u:=Q_{\lambda^{\prime}} P_{\lambda}$ and $v:=R_{\lambda^{\prime}} S_{\lambda}$ arise from (7.1) and (7.2), respectively, when we replace $\lambda$ by $\lambda^{\prime}$ and $P Q R S$ by $Q P_{\lambda} S_{\lambda} R$. Thus $a, b, c, d, h, k$ are replaced by $a^{*}, b^{*}, d, c-2 \lambda c, k^{*}, h^{*}$, respectively, hence $a^{*}, b^{*}, \lambda$ are replaced by $u, v, \lambda^{\prime}$, respectively:

$$
\begin{aligned}
& \sin d \cos u=\sin \left(d-\lambda^{\prime} d\right) \cos a^{*}+\sin \left(\lambda^{\prime} d\right) \cos h^{*}, \\
& \sin d \cos v=\sin \left(d-\lambda^{\prime} d\right) \cos b^{*}+\sin \left(\lambda^{\prime} d\right) \cos k^{*} .
\end{aligned}
$$


Inserting here the expressions for $\cos a^{*}, \cos b^{*}, \cos h^{*}, \cos k^{*}$ given by (7.1) through (7.4) leads to

$$
\begin{aligned}
& \sin c \sin d \cos u=p_{1} \cos a+p_{2} \cos k+p_{3} \cos h+p_{4} \cos b, \\
& \sin c \sin d \cos v=p_{1} \cos b+p_{2} \cos h+p_{3} \cos k+p_{4} \cos a,
\end{aligned}
$$

where

$$
\begin{array}{ll}
p_{1}=\sin (c-\lambda c) \sin \left(d-\lambda^{\prime} d\right), & p_{2}=\sin (\lambda c) \sin \left(d-\lambda^{\prime} d\right), \\
p_{3}=\sin (c-\lambda c) \sin \left(\lambda^{\prime} d\right), & p_{4}=\sin (\lambda c) \sin \left(\lambda^{\prime} d\right) .
\end{array}
$$

Now consider a comparison trapezoid $\widetilde{P} \widetilde{Q} \widetilde{R} \widetilde{S}$ for $P Q R S$, both quadrilaterals being in $S^{2}$. Then (7.5) and (7.6) likewise hold for $\widetilde{P} \widetilde{Q} \widetilde{R} \widetilde{S}$ with the same $\lambda, \lambda^{\prime}, c, d$ and hence the same $p_{1}, p_{2}, p_{3}, p_{4}$, but with $a, b$ replaced by two equal sides $\tilde{a}=\tilde{b}=a \diamond b$, and $h, k$ by two equal diagonals $\tilde{h}=\tilde{k} \geq h \diamond k$. Adding (7.5) to (7.6) for PQRS, and comparing with the same for $\widetilde{P} \widetilde{Q} \widetilde{R} \widetilde{S}$, leads after division by $\sin c \sin d$ (if $>0$ ) to

$$
\cos u+\cos v \geq \cos \tilde{u}+\cos \tilde{v}
$$

that is, $u \diamond v \leq \tilde{u}=\tilde{v}$, where $\tilde{u}:=\widetilde{P}_{\lambda} \widetilde{Q}_{\lambda^{\prime}}, \tilde{v}:=\widetilde{S}_{\lambda} \widetilde{R}_{\lambda^{\prime}}$ (they are equal because $\widetilde{P}, \widetilde{Q}$ and $\widetilde{S}, \widetilde{R}$ are symmetric about a great circle $\gamma$; cf. the last paragraph of Step 3 above). We conclude that indeed

$$
P_{\lambda} Q_{\lambda^{\prime}} \diamond S_{\lambda} R_{\lambda^{\prime}} \leq \widetilde{P}_{\lambda} \widetilde{Q}_{\lambda^{\prime}}=\widetilde{S}_{\lambda} \widetilde{R}_{\lambda^{\prime}}
$$

If $\lambda>\frac{1}{2}$, but $\lambda^{\prime} \leq \frac{1}{2}$, just replace $P Q R S$ by $Q P S R$ and $\lambda$ by $\lambda^{\prime}$. If $\lambda, \lambda^{\prime}>\frac{1}{2}$, replace $P Q R S$ by $S R Q P$ and $\lambda, \lambda^{\prime}$ by $1-\lambda, 1-\lambda^{\prime}$, both $\leq \frac{1}{2}$.

This establishes (b) in case $\sin c \sin d>0$, i.e., $c, d \in] 0, \pi[$. If, e.g., $d=0$, but $c>0$, then $a^{*}=u, b^{*}=v, h=a$, and $k=b$ in (7.1) and (7.2). We therefore arrive again at (7.7), now by adding (7.1) to (7.2) for PQRS and for $\widetilde{P} \widetilde{Q} \widetilde{R} \widetilde{S}$, and dividing by $\sin c(>0)$ while invoking $\tilde{a}=\tilde{b}=a \diamond b$ and $\tilde{h}=\tilde{k} \geq h \diamond k$.

The proof of (b) is complete, the only remaining case $c=d=0$ being trivial.

\section{Proof of Theorem 3}

By rescaling the metric $d_{Y}$ we arrange that $K=1$ and hence $R<\pi / 2$. Fix $\rho>0$ with $R+\rho<\pi / 2$, and write $B_{Y}(q, R)=B$. If $R<\pi / 4$, take $\rho=\pi / 4$, hence $R<\rho$.

Proof of (a). We employ a version of $\phi \in W^{1,2}(X, B)$ such that $\phi_{\mid X \backslash b X}$ is (Hölder) continuous (cf. [F3, Theorem 2]), and that $\phi_{\mid b X}$ is a version of $\operatorname{tr}_{b X} \phi=\operatorname{tr}_{b X} \psi \in$ $L^{2}(b X, B)$ for which $\phi_{\mid b X}$ is continuous at a given point $x_{0} \in b X$. We shall prove that $\phi: X \rightarrow B$ is continuous at $x_{0}$.

Consider a point $y \in B_{Y}(q, \rho)$; then $d_{Y}(\phi(x), y) \leq R+\rho<\pi / 2$ for $x \in X$. The function $f: X \rightarrow \mathbb{R}$ defined by

$$
f(x)=\cos d_{Y}\left(\phi\left(x_{0}\right), y\right)-\cos d_{Y}(\phi(x), y)
$$

is of class $W^{1,2}(X)$ by [EF, Corollaries 9.1, 9.2], weakly subharmonic in $X \backslash b X$ by [EF Theorem 10.2, eq. (10.19)], [F3, eq. (8.4)] (applied to $q=y$ with $R$ replaced by $R+\rho$ ), and hence subharmonic in $X \backslash b X$ by continuity; cf. footnote 7 in Section 4 above. Because $f_{\mid b X}$ is continuous at $x_{0}$ with the value 0 , it follows from the 
proof (in Section 4) of Theorem 1 (a) that (4.2) holds (this much did not involve the target $Y$, nor the map $\phi: X \rightarrow Y$, but only the subharmonicity of $f$ ); and so

$$
\limsup _{X \ni x \rightarrow x_{0}} d_{Y}(\phi(x), y) \leq d_{Y}\left(\phi\left(x_{0}\right), y\right) \text {. }
$$

If $\phi\left(x_{0}\right) \in B_{Y}(q, \rho)$, we may take $y=\phi\left(x_{0}\right)$, and it follows in that case from (8.1) that indeed $\phi(x) \rightarrow \phi\left(x_{0}\right)$ as $x \rightarrow x_{0}$ in $X$. This applies, in particular, if $R \leq \rho$ (e.g. if $R<\pi / 4$ ), so we may suppose that $R>\rho$.

In order to proceed by induction (much like it is done in [F3, pp. 397-399]), define the integer $k$ by

$$
2^{k} \rho \leq R+\rho<2^{k+1} \rho
$$

then $k>0$ because $R>\rho$. For $j \in\{0,1, \ldots, k\}$ write $\rho_{j}=2^{j} \rho$, and choose $R_{j}$ with $R_{0}=R$ so that $R_{j}+\rho_{j}$ increases strictly with $j$ from $R+\rho(<\pi / 2)$ up to

$$
R_{k}+\rho_{k}<\min \left\{\pi / 2,2 \rho_{k}\right\},
$$

and hence $R_{k}<\rho_{k}$; this is possible because $R+\rho<\pi / 2$, while $R+\rho<2 \rho_{k}$ by (8.2). It follows that

$$
R_{j}>\rho_{j} \quad \text { for } j<k ; \quad 0<R_{k}<\rho_{k} .
$$

Indeed (if $j>0$ ), $R_{j}>R+\rho-\rho_{j} \geq R+\rho-\rho_{k-1} \geq \rho_{k-1} \geq \rho_{j}$ by (8.2), and hence $R_{k}>R_{k-1}+\rho_{k-1}-\rho_{k}=R_{k-1}-\rho_{k-1}>0$.

Consider for $0<\delta \leq 1$ the image $S\left(x_{0}, \delta\right)$ of the (open) star of $x_{0}$ in $X$ under the Euclidean homothety with center $x_{0}$ and factor $\delta$; then $S\left(x_{0}, \delta\right)$ is an admissible Riemannian polyhedron 8 Suppose, for some $j \in\{0, \ldots, k\}$, that $X_{j}:=S\left(x_{0}, \delta_{j}\right)$ (with $0<\delta_{j} \leq 1$ ), $Y_{j} \subset Y$, and $q_{j} \in Y_{j}$ have been defined so that

$$
d_{Y}\left(q, q_{j}\right) \leq \rho_{j}-\rho,
$$

that $Y_{j}$ is a closed convex subset of $B \cap B_{Y}\left(q_{j}, R_{j}\right)$ (hence satisfies bipoint uniqueness, along with $B$ ), and that

$$
\phi\left(X_{j}\right) \subset Y_{j}\left(\subset B_{Y}\left(q_{j}, R_{j}\right)\right) .
$$

For $j=0$ this is fulfilled with $\delta_{0}=1, Y_{0}=B$, and $q_{0}=q$. We proceed to show that either $\phi$ is continuous at $x_{0}$, or else $j<k$ and there exist $\delta_{j+1}, Y_{j+1}, q_{j+1}$ conforming with the above requirements. In the latter case, repeat the argument with $j$ replaced by $j+1$, etc.; that will complete the inductive proof that $\phi$ is indeed continuous at $x_{0}$.

For the given index $j \in\{0, \ldots, k\}$ let $X_{j}, Y_{j}, q_{j}, R_{j}, \rho_{j}$ take the place of $X, B, q$, $R, \rho$, noting that $R_{j}+\rho_{j}<\pi / 2$ by (8.3). Then (8.1) remains in force (with the corresponding proof) for any $y \in B_{Y}\left(q_{j}, \rho_{j}\right)$ (in place of $y \in B_{Y}(q, \rho)$ ) because $d_{Y}(\phi(x), y) \leq R_{j}+\rho_{j}<\pi / 2$ for $x \in X_{j}$.

If $\phi\left(x_{0}\right) \in B_{Y}\left(q_{j}, \rho_{j}\right)$, this shows that $\phi$ is continuous at $x_{0}$. That applies if $j=k$ since then $R_{j}<\rho_{j}$ by (8.4), and so $\phi\left(x_{0}\right) \in \phi\left(X_{j}\right) \subset B_{Y}\left(q_{j}, \rho_{j}\right)$ by (8.6).

We may thus assume that $j<k$, and hence $R_{j}>\rho_{j}$ by (8.4). It follows by (8.5) and (8.2) that $d_{Y}\left(q, q_{j}\right) \leq \rho_{j}-\rho \leq R-\rho_{j}$ because $2 \rho_{j}=\rho_{j+1} \leq \rho_{k}$. Thus $B_{Y}\left(q_{j}, \rho_{j}\right) \subset B$, and so $B_{Y}\left(q_{j}, \rho_{j}\right)$ is convex (hence satisfies bipoint uniqueness, along with $B)$. If $\phi$ is discontinuous at $x_{0}$, then $\phi\left(x_{0}\right) \notin B_{Y}\left(q_{j}, \rho_{j}\right)$, by (8.1) as noted above. Define $q_{j+1}$ as the nearest point projection of $\phi\left(x_{0}\right)$ on the convex

\footnotetext{
${ }^{8}$ In [F3, p. 398] the star of $a$ in $X$ should be open (not closed, as stated-an error of no consequence).
} 
ball $B_{Y}\left(q_{j}, \rho_{j}\right)$; thus $q_{j+1}$ is the point of the geodesic segment $q_{j} \phi\left(x_{0}\right)$ at distance $\rho_{j}$ from $q_{j}$, and so $d_{Y}\left(q, q_{j+1}\right) \leq d_{Y}\left(q, q_{j}\right)+\rho_{j} \leq \rho_{j+1}-\rho$, as required; cf. (8.5). Furthermore, by (8.6),

$$
d_{Y}\left(\phi\left(x_{0}\right), q_{j+1}\right)=d_{Y}\left(\phi\left(x_{0}\right), q_{j}\right)-\rho_{j} \leq R_{j}-\rho_{j}=R_{j}+\rho_{j}-\rho_{j+1}<R_{j+1},
$$

and hence

$$
\phi\left(x_{0}\right) \in Y_{j+1}:=Y_{j} \cap B_{Y}\left(q_{j+1}, R_{j+1}\right)=B_{Y_{j}}\left(q_{j+1}, R_{j+1}\right) .
$$

This closed ball $Y_{j+1}$ in $Y_{j}$ of radius $R_{j+1}<\pi / 2-\rho_{j+1}$, by (8.3), is a convex subset of $Y_{j}$ and hence of $B$, satisfying bipoint uniqueness. We may now apply (8.1) to $y=q_{j+1}\left(\in B_{Y}\left(q_{j}, \rho_{j}\right)\right)$, and so (8.7) further implies

$$
\limsup _{X \ni x \rightarrow x_{0}} d_{Y}\left(\phi(x), q_{j+1}\right) \leq d_{Y}\left(\phi\left(x_{0}\right), q_{j+1}\right)<R_{j+1} ;
$$

hence there does exist $\delta_{j+1}$ with $0<\delta_{j+1} \leq \delta_{j}$ such that $X_{j+1}:=S\left(x_{0}, \delta_{j+1}\right)$ satisfies $\phi\left(X_{j+1}\right) \subset Y_{j+1}$, thus extending (8.6) and completing the inductive proof that $\phi$ is indeed continuous at $x_{0}$.

Proof of (b). We choose here not to pass to the map $\phi_{0} \times \phi_{1}$ into $Y \times Y$ (as it was done in the proof of Theorem 1) because that would require assuming $R \sqrt{2}<\pi / 2$, rather than just $R<\pi / 2$; cf. (4.3). Instead, Lemma 2 provides a similar symmetry.

Replacing $X \backslash b X$ by any admissible Riemannian polyhedron $(X, g)$ (possibly noncompact) we show more generally that $\mathcal{L} \theta \geq 0$ holds in $X$ (in the weak sense), cf. (2.6), (2.7), now with $K=1$, for any two harmonic maps $\phi_{0}, \phi_{1}:(X, g) \rightarrow\left(Y, d_{Y}\right)$, or equivalently: two continuous locally $E$-minimizing maps [EF Remark 12.1]. As explained after (4.7), $u$ and hence $\theta$ are of class $W_{\mathrm{loc}}^{1,2}(X)$.

Given a point $x_{0} \in X$ we shall prove that $\mathcal{L} \theta \geq 0$ holds in some open neighbourhood $U$ of $x_{0}$ in $X$. Because $\theta$ is continuous and $\geq 0$, we may assume that $\theta\left(x_{0}\right)>0$, that is, $\phi_{0}\left(x_{0}\right) \neq \phi_{1}\left(x_{0}\right)$ (cf. text following (2.7)). Choose $U$ as in the definition of a local $E$-minimizer (Section 1), in this case $\phi_{0}$, resp. $\phi_{1}$, and furthermore so that $x_{0} \in U$ and, for some constant $\sigma>0$,

$$
u(x):=d_{Y}\left(\phi_{0}(x), \phi_{1}(x)\right)>\sigma, \quad x \in U .
$$

With $0<\rho<\pi / 2-R$ as before, let $\epsilon>0$ be small enough so that, for all $x \in U$ and $x^{\prime} \in B_{U}(x, \epsilon)$,

$$
d_{i}:=d_{Y}\left(\phi_{i}(x), \phi_{i}\left(x^{\prime}\right)\right)<\rho, \quad i=0,1,
$$

$\phi_{i}$ being uniformly continuous on $U$. For such $x, x^{\prime}$ apply Lemma 2 to the (ordered) quadrilateral

$$
P Q R S=\phi_{0}(x) \phi_{0}\left(x^{\prime}\right) \phi_{1}\left(x^{\prime}\right) \phi_{1}(x)
$$

in $B$, in which the side lengths $u=u(x)=P S$ and $u^{\prime}=u\left(x^{\prime}\right)=Q R$ are $\leq 2 R<$ $\pi-2 \rho$; and so $2\left(d_{0} \diamond d_{1}\right)+u+u^{\prime}<2 \pi$; cf. (2.3). By Lemma 2 (a) there exists in $S^{2}$ a comparison trapezoid $\widetilde{P} \widetilde{Q} \widetilde{R} \widetilde{S}$ for $P Q R S$, in the sense defined in Section 2 before that lemma; in particular,

$$
\begin{aligned}
u & =d_{Y}\left(\phi_{0}(x), \phi_{1}(x)\right)=P S=\widetilde{P} \widetilde{S}, \\
u^{\prime} & =d_{Y}\left(\phi_{0}\left(x^{\prime}\right), \phi_{1}\left(x^{\prime}\right)\right)=Q R=\widetilde{Q} \widetilde{R} .
\end{aligned}
$$

For any continuous function $\kappa$ with support in the above set $U$ such that $0 \leq \kappa \leq 1$ we have (after restricting $\phi_{0}$ and $\phi_{1}$ to $U$ )

$$
E\left(\phi_{\kappa}\right) \geq E\left(\phi_{0}\right), \quad E\left(\phi_{1-\kappa}\right) \geq E\left(\phi_{1}\right),
$$


the maps $\phi_{\kappa}, \phi_{1-\kappa}: U \rightarrow B$ (competing with $\phi_{0}$, resp. $\left.\phi_{1}\right)$ being defined by

$$
\begin{aligned}
\phi_{\kappa}(x) & =(1-\kappa(x)) \phi_{0}(x)+\kappa(x) \phi_{1}(x), \\
\phi_{1-\kappa}(x) & =(1-\kappa(x)) \phi_{1}(x)+\kappa(x) \phi_{0}(x),
\end{aligned}
$$

with the usual abuse of notation. Write briefly $\kappa(x)=\kappa, \kappa\left(x^{\prime}\right)=\kappa^{\prime}$ for $x \in U$, $x^{\prime} \in B_{U}(x, \epsilon)$, and consider in $S^{2}$ the points

$$
\widetilde{P}_{\kappa}=(1-\kappa) \widetilde{P}+\kappa \widetilde{S}, \quad \widetilde{Q}_{\kappa^{\prime}}=\left(1-\kappa^{\prime}\right) \widetilde{Q}+\kappa^{\prime} \widetilde{R} .
$$

By extending (8.9) write

$$
\begin{aligned}
d_{\kappa} & =d_{Y}\left(\phi_{\kappa}(x), \phi_{\kappa^{\prime}}\left(x^{\prime}\right)\right), \\
d_{1-\kappa} & =d_{Y}\left(\phi_{1-\kappa}(x), \phi_{1-\kappa^{\prime}}\left(x^{\prime}\right)\right),
\end{aligned}
$$

where of course $\phi_{\kappa^{\prime}}\left(x^{\prime}\right):=\left(1-\kappa\left(x^{\prime}\right)\right) \phi_{0}\left(x^{\prime}\right)+\kappa\left(x^{\prime}\right) \phi_{1}\left(x^{\prime}\right)$, etc. By Lemma $2(\mathrm{~b})$, $d_{\kappa} \diamond d_{1-\kappa} \leq \widetilde{P}_{\kappa} \widetilde{Q}_{\kappa^{\prime}}$, and since $d_{0} \diamond d_{1}=\widetilde{P} \widetilde{Q}$ we obtain in view of $(2.3)$

$$
\frac{1}{2}\left(\cos d_{0}-\cos d_{\kappa}\right)+\frac{1}{2}\left(\cos d_{1}-\cos d_{1-\kappa}\right) \leq \cos \widetilde{P} \widetilde{Q}-\cos \widetilde{P}_{\kappa} \widetilde{Q}_{\kappa^{\prime}} .
$$

The great circles through $\widetilde{P}$ and $\widetilde{S}$, resp. $\widetilde{Q}$ and $\widetilde{R}$, meet in at least one point $\widetilde{O}$ of $S^{2}$ with distance $\leq \pi / 2$ from $\widetilde{R}$ and $\widetilde{S}$. Write

$$
\begin{gathered}
d:=d_{0} \diamond d_{1}=\widetilde{P} \widetilde{Q}, \\
v:=\widetilde{O} \widetilde{P}=\frac{1}{2}(\pi+u), \quad v^{\prime}:=\widetilde{O} \widetilde{Q}=\frac{1}{2}\left(\pi+u^{\prime}\right) ;
\end{gathered}
$$

cf. (8.10). Then $v, v^{\prime} \in\left[\frac{1}{2}(\pi+\sigma), \pi-\rho\right]$ (cf. (8.8)), and so $\cot v, \cot v^{\prime}<0$, marking an important difference from the proof of [EF, Theorem 10.2], [F3, Theorem 2].

Given a function $\lambda \in \operatorname{Lip}_{c}(U), 0 \leq \lambda \leq \sigma / \pi$, apply the above to

$$
\kappa=\lambda v / u, \quad \kappa^{\prime}=\lambda^{\prime} v^{\prime} / u^{\prime}
$$

for $x \in U, x^{\prime} \in B_{U}(x, \epsilon)$; here $\lambda=\lambda(x), \lambda^{\prime}=\lambda\left(x^{\prime}\right)$. Then $0 \leq \kappa \leq 1$ because $v<\pi$ and $u>\sigma$, by (8.8). Furthermore, $\widetilde{P} \widetilde{P}_{\kappa}=\kappa u=\lambda \widetilde{O} \widetilde{P}$ and $\widetilde{Q} \widetilde{Q}_{\kappa^{\prime}}=\kappa^{\prime} u^{\prime}=\lambda{ }^{\prime} \widetilde{O} \widetilde{Q}$. From the spherical cosine relation, applied to the triangles $\widetilde{O} \widetilde{P} \widetilde{Q}$ and $\widetilde{O} \widetilde{P}_{\kappa} \widetilde{Q}_{\kappa^{\prime}}$, we therefore obtain (cf. [EF, p. 193]), after eliminating the common angle at $\widetilde{O}$,

$$
\begin{aligned}
\sin v \sin v^{\prime} \cos \widetilde{P}_{\kappa} \widetilde{Q}_{\kappa^{\prime}} & =\sin (v-\lambda v) \sin \left(v^{\prime}-\lambda^{\prime} v^{\prime}\right) \cos d \\
& +\sin (v-\lambda v) \sin \left(\lambda^{\prime} v^{\prime}\right) \cos v \\
& +\sin v^{\prime} \sin (\lambda v) \cos \left(v^{\prime}-\lambda^{\prime} v^{\prime}\right) .
\end{aligned}
$$

Insert this expression for $\cos \widetilde{P}_{\kappa} \widetilde{Q}_{\kappa^{\prime}}$ in (8.14), together with $\cos \widetilde{P} \widetilde{Q}=\cos d=$ $\frac{1}{2}\left(\cos d_{0}+\cos d_{1}\right)$ from (8.15). After some manipulations serving to make (1.2), (1.4), and (1.5) applicable this leads to

$$
\frac{1}{2}\left(\cos d_{0}-\cos d_{\kappa}\right)+\frac{1}{2}\left(\cos d_{1}-\cos d_{1-\kappa}\right) \leq R^{(1)}+R^{(2)}+R^{(3)}+R^{(4)} ;
$$

cf. [EF, eq. (10.17)], [F3, eq. (8.2)]. Here

$$
\begin{aligned}
R^{(1)}: & =-2 \sin ^{2}\left(\frac{1}{2} d\right)\left(1-\frac{\sin (v-\lambda v)}{\sin v} \frac{\sin \left(v^{\prime}-\lambda^{\prime} v^{\prime}\right)}{\sin v^{\prime}}\right) \\
& =-2 \sin ^{2}\left(\frac{1}{2} d\right)\left(\lambda v \cot v+\lambda^{\prime} v^{\prime} \cot v^{\prime}+O\left(\lambda^{2}+\lambda^{\prime 2}\right)\right) \\
& =-\frac{1}{4}\left(d_{0}^{2}+d_{1}^{2}\right)\left(2 \lambda v \cot v+\omega(\epsilon)+O\left(\lambda^{2}+\lambda^{\prime 2}\right)\right),
\end{aligned}
$$


where $\omega(\epsilon)$ stands for any term tending to 0 as $\epsilon \rightarrow 0$, uniformly for $x \in U$ and $x^{\prime} \in$ $B_{U}(x, \epsilon)$. Indeed, by $(2.3),(8.9)$, and (8.15), $2 \sin ^{2}\left(\frac{1}{2} d\right)=\sin ^{2}\left(\frac{1}{2} d_{0}\right)+\sin ^{2}\left(\frac{1}{2} d_{1}\right)=$ $\frac{1}{4}\left(d_{0}^{2}+d_{1}^{2}\right)(1+\omega(\epsilon))$; furthermore, $-\cot v \in[\tan \sigma / 2, \cot \rho]$; and $\phi_{0}, \phi_{1}, \kappa, \lambda$, and $v$ are uniformly continuous. As in [EF, p. 193] and [F3, p. 394],

$$
\begin{aligned}
R^{(2)}: & =\cos (\lambda v) \cos \left(\lambda^{\prime} v^{\prime}\right)\left(\cos v-\cos v^{\prime}\right)\left(\frac{\tan (\lambda v)}{\sin v}-\frac{\tan \left(\lambda^{\prime} v^{\prime}\right)}{\sin v^{\prime}}\right) \\
& =\left(\cos v-\cos v^{\prime}\right)\left(\frac{\tan (\lambda v)}{\sin v}-\frac{\tan \left(\lambda^{\prime} v^{\prime}\right)}{\sin v^{\prime}}\right)\left(1+O\left(\lambda^{2}+\lambda^{\prime 2}\right)\right) ; \\
R^{(3)}: & =\left(\cos v-\cos v^{\prime}\right)^{2} \frac{\sin (\lambda v)}{\sin v} \frac{\sin \left(\lambda^{\prime} v^{\prime}\right)}{\sin v^{\prime}} \\
& =\left(\cos v-\cos v^{\prime}\right)^{2} O\left(\lambda^{2}+\lambda^{\prime 2}\right) ; \\
R^{(4)}: & =2 \sin ^{2} \frac{\lambda v-\lambda^{\prime} v^{\prime}}{2}-2 \sin ^{2} \frac{v-v^{\prime}}{2} \frac{\sin (\lambda v)}{\sin v} \frac{\sin \left(\lambda^{\prime} v^{\prime}\right)}{\sin v^{\prime}} \\
& \leq \frac{1}{2}\left(\lambda v-\lambda^{\prime} v^{\prime}\right)^{2} .
\end{aligned}
$$

The function $v=\frac{1}{2}(\pi+u)$ from (8.16) with values in $\left[\frac{1}{2}(\pi+\sigma), \pi-\rho\right]$ is of class $W_{\text {loc }}^{1,2}(U)$, like $u=d_{Y}\left(\phi_{0}, \phi_{1}\right)$ (cf. text following (4.7)), and so are therefore $v \cot v, \cos v, \lambda v, \tan (\lambda v) / \sin v$, and $\sin (\lambda v) / \sin v$, occurring in the final estimates of $R^{(1)}$ through $R^{(4)}$, according to $\mathrm{EF}$, Lemma 5.2]; noting that, for example, $\tan (\lambda v) / \sin v=\lambda[\tan (\lambda v) /(\lambda v)][v / \sin v]$ and that the product of two bounded $W^{1,2}$-functions is a bounded $W^{1,2}$-function. Furthermore, $\phi_{\kappa}=\phi_{0}, \phi_{1-\kappa}=\phi_{1}$ in $U \backslash \operatorname{supp} \lambda$; cf. (8.12).

We proceed to estimate $d_{\kappa} \diamond d_{1-\kappa}\left(\leq \widetilde{P}_{\kappa} \widetilde{Q}_{\kappa^{\prime}}\right)$ from above in terms of $d_{0} \diamond d_{1}=d$. The spherical cosine relation applied to $\widetilde{P} \widetilde{Q} \widetilde{O}$ with angle $\theta$ at $\widetilde{O}$ may be written (after inserting (8.15), (8.16)):

$$
\sin ^{2} \frac{d}{2}=\sin ^{2} \frac{v-v^{\prime}}{2}+\sin v \sin v^{\prime} \sin ^{2} \frac{\theta}{2} .
$$

Consider along with $\widetilde{Q}_{\kappa^{\prime}}$ the auxiliary point $\widetilde{Q}_{\kappa}:=(1-\kappa(x)) \widetilde{Q}+\kappa(x) \widetilde{R}$. Replacing $\widetilde{P}, \widetilde{Q}$ by $\widetilde{P}_{\kappa}, \widetilde{Q}_{\kappa}$ amounts to replacing $v, v^{\prime}$ by $v-\lambda v, v^{\prime}-\lambda v^{\prime}$ (where $\lambda=\lambda(x)=$ $\kappa(x) u(x) / v(x))$, while keeping $\theta$. Inserting $\sin ^{2}\left[\frac{1}{2}(1-\lambda)\left(v-v^{\prime}\right)\right] \leq \sin ^{2}\left[\frac{1}{2}\left(v-v^{\prime}\right)\right]$ (valid because $\left|v-v^{\prime}\right|=\frac{1}{2}\left|u-u^{\prime}\right|<\frac{1}{2} \pi$ by (8.16)), $\sin (v-\lambda v) \sin \left(v^{\prime}-\lambda v^{\prime}\right) \leq 1$, and $\sin v \sin v^{\prime} \geq \sin ^{2} \rho$, leads after elimination of $\theta$ to

$$
\sin ^{2}\left(\frac{1}{2} \widetilde{P}_{\kappa} \widetilde{Q}_{\kappa}\right) \leq \frac{\sin ^{2}\left(\frac{1}{2} d\right)}{\sin ^{2} \rho}
$$

and hence $\widetilde{P}_{\kappa} \widetilde{Q}_{\kappa}=O(d)=O\left(\left(d_{0}^{2}+d_{1}^{2}\right)^{1 / 2}\right)$. Combining this with $\widetilde{Q}_{\kappa} \widetilde{Q}_{\kappa^{\prime}}=$ $\left|\lambda-\lambda^{\prime}\right| v^{\prime} \leq \pi\left|\lambda(x)-\lambda\left(x^{\prime}\right)\right|$ and $d_{\kappa}, d_{1-\kappa} \leq 2\left(d_{\kappa} \diamond d_{1-\kappa}\right)$ leads to

$$
\begin{aligned}
d_{\kappa}^{2}+d_{1-\kappa}^{2} & \leq 8\left(d_{\kappa} \diamond d_{1-\kappa}\right)^{2} \leq 8 \widetilde{P}_{\kappa} \widetilde{Q}_{\kappa^{\prime}}^{2} \leq 16\left(\widetilde{P}_{\kappa} \widetilde{Q}_{\kappa}^{2}+\widetilde{Q}_{\kappa} \widetilde{Q}_{\kappa^{\prime}}^{2}\right) \\
& =O\left(d_{0}^{2}+d_{1}^{2}+\left|\lambda(x)-\lambda\left(x^{\prime}\right)\right|^{2}\right)=\omega(\epsilon)
\end{aligned}
$$

by uniform continuity of $\phi_{0}, \phi_{1}, \lambda$ on $U$. It follows that

$$
d_{\kappa}^{4}+d_{1-\kappa}^{4}=\omega(\epsilon) O\left(d_{0}^{2}+d_{1}^{2}+\left|\lambda(x)-\lambda\left(x^{\prime}\right)\right|^{2}\right) .
$$

Note that $1-\cos d_{\kappa}=2 \sin ^{2}\left(\frac{1}{2} d_{\kappa}\right)=2 \tan ^{2}\left(\frac{1}{2} d_{\kappa}\right)\left(1-\sin ^{2}\left(\frac{1}{2} d_{\kappa}\right)\right) \geq \frac{1}{2} d_{\kappa}^{2}\left(1-\frac{1}{4} d_{\kappa}^{2}\right)$, and on the other hand, $1-\cos d_{0} \leq \frac{1}{2} d_{0}^{2}$. Hence

$$
\frac{1}{2}\left(d_{\kappa}^{2}-d_{0}^{2}\right) \leq \frac{1}{8} d_{\kappa}^{4}+\left(\cos d_{0}-\cos d_{\kappa}\right) .
$$


Adding to this the corresponding inequality with $\kappa$ replaced by $1-\kappa$, and 0 by 1 , we obtain for $f \in C_{c}(X,[0,1])$ after dividing by $2 \epsilon^{m+2}$ and invoking (1.1), (1.3), $(2.3)$, and (8.17),

$$
\begin{aligned}
& \frac{1}{4} \limsup _{\epsilon \rightarrow 0} \int_{U}\left(e_{\epsilon}\left(\phi_{\kappa}\right)-e_{\epsilon}\left(\phi_{0}\right)+e_{\epsilon}\left(\phi_{1-\kappa}\right)-e_{\epsilon}\left(\phi_{1}\right)\right) f d \mu \\
& \leq \limsup _{\epsilon \rightarrow 0} \int_{U} f(x) d \mu(x) \int_{B_{U}(x, \epsilon)} \frac{1}{\epsilon^{m+2}} \sum_{j=1}^{4} R^{(j)} d \mu\left(x^{\prime}\right),
\end{aligned}
$$

the contribution from $d_{\kappa}^{4}+d_{1-\kappa}^{4}($ cf. (8.19)) being

$$
\limsup _{\epsilon \rightarrow 0}\left[\omega(\epsilon) O\left(\int_{U}\left[\sum_{i=0}^{1} e_{\epsilon}\left(\phi_{i}\right)(x)+\int_{B_{U}(x, \epsilon)} \frac{\left|\lambda(x)-\lambda\left(x^{\prime}\right)\right|^{2}}{\epsilon^{m+2}} d \mu\left(x^{\prime}\right)\right] f(x) d \mu(x)\right)\right]=0
$$

according to (1.1), the integral over $U$ having a finite limit for $\epsilon \rightarrow 0$, by (1.3) and (1.5).

Inserting again $\lambda^{\prime}=\lambda+O(\epsilon)$ in the remainder terms in the above estimates of $R^{(1)}, R^{(2)}, R^{(3)}$, and $R^{(4)}$, to be inserted in (8.20), we obtain by application of (1.2) through (1.6), and (8.11),

$$
\begin{aligned}
0 \leq & \frac{1}{4}\left(E\left(\phi_{\kappa}\right)-E\left(\phi_{0}\right)\right)+\frac{1}{4}\left(E\left(\phi_{1-\kappa}\right)-E\left(\phi_{1}\right)\right) \\
= & \frac{1}{4} \lim _{f \in C_{c}(U,[0,1])} \limsup _{\epsilon \rightarrow 0} \int_{U}\left(e_{\epsilon}\left(\phi_{\kappa}\right)-e_{\epsilon}\left(\phi_{0}\right)+e_{\epsilon}\left(\phi_{1-\kappa}\right)-e_{\epsilon}\left(\phi_{1}\right)\right) f d \mu \\
\leq & \limsup _{f \in C_{c}(U,[0,1])} \limsup _{\epsilon \rightarrow 0} \int_{U} f(x) d \mu(x) \int_{B_{U}(x, \epsilon)} \frac{1}{\epsilon^{m+2}} \sum_{j=1}^{4} R^{(j)} d \mu\left(x^{\prime}\right) \\
\leq & -\int_{U} \frac{\lambda}{2}\left(e\left(\phi_{0}\right)+e\left(\phi_{1}\right)\right) v \cot v d \mu \\
& +c_{m} \int_{U}\left\langle\nabla \cos v, \nabla \frac{\tan (\lambda v)}{\sin v}\right\rangle d \mu+\frac{1}{2} c_{m} \int_{U}|\nabla(\lambda v)|^{2} d \mu+O\left(\|\lambda\|_{L^{\infty}}^{2}\right) .
\end{aligned}
$$

Concerning the first term after the last inequality sign, note that (1.3) is applied here with $f$ replaced by $-\frac{1}{2} f \lambda v \cot v(\geq 0)$.

For given $\lambda \in \operatorname{Lip}_{c}^{+}(U)$ apply the resulting inequality with $t \lambda$ in place of $\lambda$ for small $t>0$, divide by $t$, and let $t \rightarrow 0$, noting that

$$
\nabla \frac{\tan (t \lambda v)}{t \sin v} \rightarrow \nabla \frac{\lambda v}{\sin v} \quad \text { in } L^{2}(U) \text { as } t \rightarrow 0
$$

This leads to

$$
\int_{U}\left\langle\nabla \cos v, \nabla \frac{\lambda v}{\sin v}\right\rangle d \mu \geq \frac{1}{2 c_{m}} \int_{U} \lambda v \cot v \sum_{i=0}^{1} e\left(\phi_{i}\right) d \mu, \quad \lambda \in \operatorname{Lip}_{c}^{+}(U) .
$$

As in [EF, eq. (10.18)], $\lambda v / \sin v$ can be replaced here by any function $\lambda \in \operatorname{Lip}_{c}^{+}(U)$ :

$$
\int_{U}\langle\nabla \cos v, \nabla \lambda\rangle d \mu \geq \frac{1}{2 c_{m}} \int_{U} \lambda \cos v \sum_{i=0}^{1} e\left(\phi_{i}\right) d \mu .
$$


Inserting also $v=\frac{1}{2}(\pi+u)$ from (8.16), with $u=d_{Y}\left(\phi_{0}, \phi_{1}\right)$ from (8.8), we finally obtain

$$
\int_{U}\left\langle\nabla \sin \frac{u}{2}, \nabla \lambda\right\rangle d \mu \leq \frac{1}{2 c_{m}} \int_{U} \lambda \sin \frac{u}{2} \sum_{i=0}^{1} e\left(\phi_{i}\right) d \mu, \quad \lambda \in \operatorname{Lip}_{c}^{+}(U) ;
$$

that is, in the weak sense in $U$,

$$
\Delta \sin \frac{u}{2} \geq-\frac{1}{2 c_{m}} \sin \frac{u}{2} \sum_{i=0}^{1} e\left(\phi_{i}\right)
$$

On the other hand, the functions $v_{i}:=d_{Y}\left(\phi_{i}, q\right), i=0,1$, satisfy the analogue of (8.21):

$$
\int_{U}\left\langle\nabla \cos v_{i}, \nabla \lambda\right\rangle d \mu \geq \frac{1}{c_{m}} \int_{U} \lambda \cos v_{i} e\left(\phi_{i}\right) d \mu, \quad \lambda \in \operatorname{Lip}_{c}^{+}(U),
$$

or in the weak sense

$$
\Delta \cos v_{i} \leq-\frac{1}{c_{m}} \cos v_{i} e\left(\phi_{i}\right)
$$

cf. [JK, Lemma 4, eq. (29)] and [Se2, Corollary (2.10)] (invoking [F4 Proposition 1]). Indeed, (8.23) is a slightly, but importantly sharper version of [EF eq. (10.19)], [F3, eq. (8.5)] (applied now to $\phi_{i}, v_{i}$ in place of $\phi, v$ ). It holds because $\phi_{i}$ is continuous here, by hypothesis; and that allows for the same sharper estimate of $R^{(1)}$ as in (8.18) instead of the final estimate of $R_{1}$ in [EF, p. 193], [F3, §8]; besides, we no longer estimate $v^{-1} \sin v$ from below, as it was done (of necessity) in [EF, line preceding (10.19)] and in [F3, (8.4)] $]^{\mathrm{g}}$

We are now prepared to prove that the Jäger-Kaul function

$$
\theta=\theta\left(\phi_{0}, \phi_{1}\right)=\frac{1-\cos u}{\cos v_{0} \cos v_{1}}
$$

with $u=d_{Y}\left(\phi_{0}, \phi_{1}\right), v_{i}=d_{Y}\left(\phi_{i}, q\right), i=0,1$, does satisfy

$$
\mathcal{L}(\theta):=\operatorname{div}\left(\cos v_{0} \cos v_{1} \nabla \theta\right) \geq 0
$$

in the weak sense in $U$ (cf. (2.6), (2.7) now with $K=1$ ); that is,

$$
\int_{U} \cos v_{0} \cos v_{1}\langle\nabla \theta, \nabla \lambda\rangle d \mu \leq 0, \quad \lambda \in \operatorname{Lip}_{c}^{+}(U) .
$$

Because $\operatorname{Lip}_{c}^{+}(U)$ is dense in $W_{0}^{1,2}(U)^{+}$EF Proposition $\left.5.1(\mathrm{~d})\right]$, we may replace $\operatorname{Lip}_{c}^{+}(U)$ by $W_{c}^{1,2}(U)^{+}$in (8.21), (8.22), (8.23), and (8.25). From (8.24) we obtain, inserting $1-\cos u=2 \sin ^{2}\left(\frac{1}{2} u\right)$, and using elementary facts from [EF, Chapter 5$]$,

$$
\cos v_{0} \cos v_{1} \nabla \theta=4 \sin \frac{u}{2} \nabla \sin \frac{u}{2}-2 \sin ^{2} \frac{u}{2} \sum_{i=0}^{1} \frac{\nabla \cos v_{i}}{\cos v_{i}} .
$$

\footnotetext{
${ }^{9}$ Actually, it was shown afterwards in [EF, Theorem 10.2], [F3. Theorem 2], that $\phi$ (in that setting) is Hölder continuous, and so [EF, (10.19)] and [F3] (8.4)] can be replaced at the end by what corresponds to (8.21) above.
} 
Inserting this in the left-hand member of (8.25) gives in the first place

$$
\begin{aligned}
4 \int_{U} \sin & \frac{u}{2}\left\langle\nabla \sin \frac{u}{2}, \nabla \lambda\right\rangle d \mu \\
& =4 \int_{U}\left\langle\nabla \sin \frac{u}{2}, \nabla\left(\lambda \sin \frac{u}{2}\right)\right\rangle d \mu-4 \int_{U}\left|\nabla \sin \frac{u}{2}\right|^{2} \lambda d \mu \\
& \leq \frac{2}{c_{m}} \int_{U} \lambda \sin ^{2} \frac{u}{2} \sum_{i=0}^{1} e\left(\phi_{i}\right) d \mu-4 \int_{U}\left|\nabla \sin \frac{u}{2}\right|^{2} \lambda d \mu
\end{aligned}
$$

for $\lambda \in \operatorname{Lip}_{c}^{+}(U)$, by (8.22) with $\lambda$ replaced by $\lambda \sin (u / 2) \in W_{c}^{1,2}(U)^{+}$. Secondly,

$$
\begin{aligned}
-2 \int_{U} \sin ^{2} & \frac{u}{2} \sum_{i=0}^{1} \frac{1}{\cos v_{i}}\left\langle\nabla \cos v_{i}, \nabla \lambda\right\rangle d \mu \\
= & -2 \int_{U} \sum_{i=0}^{1}\left\langle\nabla \cos v_{i}, \nabla\left(\frac{1}{\cos v_{i}} \lambda \sin ^{2} \frac{u}{2}\right)\right\rangle d \mu \\
& +2 \int_{U} \sum_{i=0}^{1}\left\langle\nabla \cos v_{i}, \nabla\left(\frac{1}{\cos v_{i}} \sin ^{2} \frac{u}{2}\right)\right\rangle \lambda d \mu \\
\leq & -\frac{2}{c_{m}} \int_{U} \lambda \sin ^{2} \frac{u}{2} \sum_{i=0}^{1} e\left(\phi_{i}\right) d \mu-2 \int_{U} \sum_{i=0}^{1}\left|\frac{\nabla \cos v_{i}}{\cos v_{i}}\right|^{2} \sin ^{2} \frac{u}{2} \lambda d \mu \\
& +4 \int_{U} \sum_{i=0}^{1}\left\langle\frac{\nabla \cos v_{i}}{\cos v_{i}}, \nabla \sin \frac{u}{2}\right\rangle \sin \frac{u}{2} \lambda d \mu
\end{aligned}
$$

for $\lambda \in \operatorname{Lip}_{c}^{+}(U)$, by (8.23) with $\lambda$ replaced by $\left(1 / \cos v_{i}\right) \lambda \sin ^{2}(u / 2) \in W_{c}^{1,2}(U)^{+}$, followed by summation over $i=1,2$. Adding (8.27) to (8.28) while invoking (8.26) leads to $(8.25)$ because the terms containing $\sum_{i=0}^{1} e\left(\phi_{i}\right)$ cancel and

$$
\begin{aligned}
& 4\left|\nabla \sin \frac{u}{2}\right|^{2}+2 \sin ^{2} \frac{u}{2} \sum_{i=0}^{1}\left|\frac{\nabla \cos v_{i}}{\cos v_{i}}\right|^{2}-4 \sin \frac{u}{2}\left\langle\nabla \sin \frac{u}{2}, \sum_{i=0}^{1} \frac{\nabla \cos v_{i}}{\cos v_{i}}\right\rangle \\
& =\left|2 \nabla \sin \frac{u}{2}-\sin \frac{u}{2} \sum_{i=0}^{1} \frac{\nabla \cos v_{i}}{\cos v_{i}}\right|^{2}+\sin ^{2} \frac{u}{2}\left|\frac{\nabla \cos v_{0}}{\cos v_{0}}-\frac{\nabla \cos v_{1}}{\cos v_{1}}\right|^{2} \geq 0 .
\end{aligned}
$$

\section{Proof of Part (b) of Proposition 2}

For given $r>0$ write $\theta_{r}(t)=\theta(t)=r / \sqrt{r^{2}+t^{2}}, 0 \leq t<\infty$. Then $\theta(0)=1$, $\theta(t) \leq 1, t \mapsto \theta(t)$ is decreasing, and $t \mapsto t \theta(t)$ is increasing and bounded: $t \theta(t) \leq r$.

Fix a point $q \in N$. By the hypotheses on $N$ the exponential map $\exp _{q}: T_{q}(N) \rightarrow N$ is bijective, so Euclidean coordinates $y^{\alpha}$ on $T_{q}(N)$ serve as normal coordinates on $N$, centered at $q$. Identify $N$ with $T_{q}(N)=\mathbb{R}^{n}$ via $\left(\exp _{q}\right)^{-1}$ and these Euclidean coordinates $y^{\alpha}$; then $d_{N}^{2}(q, y)=|y|^{2}=\sum_{\alpha=1}^{n}\left(y^{\alpha}\right)^{2}$. For $y \in N$ and $c \in \mathbb{R}, c y \in Y$ is thus well defined and has coordinates $c y^{\alpha}$. In particular, we define $p_{\theta}(y)=\theta(|y|) y \in$ $N$ for $y \in N$; thus $\left|p_{\theta}(y)\right|=\theta(|y|)|y| \leq r$.

We first show by triangle comparison that the map $p_{\theta}: N \rightarrow N$ is contractive:

$$
d_{N}\left(p_{\theta}(y), p_{\theta}\left(y^{\prime}\right)\right) \leq d_{N}\left(y, y^{\prime}\right), \quad y, y^{\prime} \in N .
$$


Let a triangle $0 \tilde{y} \tilde{y}^{\prime}$ in $\mathbb{R}^{2}$ have the same side lengths as the triangle $q y y^{\prime}$ in $N$. If e.g. $|y| \leq\left|y^{\prime}\right|$, then

$$
\left|p_{\theta}(y)\right|=\theta(|y|)|y| \leq \theta\left(\left|y^{\prime}\right|\right)\left|y^{\prime}\right|=\left|p_{\theta}\left(y^{\prime}\right)\right|
$$

since $t \theta(t)$ is increasing. The point $z:=\left[\theta\left(\left|y^{\prime}\right|\right) / \theta(|y|)\right] y^{\prime}$ lies on $\left[q, y^{\prime}\right]$ because $\theta$ is decreasing; thus $|y| \leq|z| \leq\left|y^{\prime}\right|$, by the inequality in (9.2). This translates into $|\tilde{y}| \leq|\tilde{z}| \leq\left|\tilde{y}^{\prime}\right|$. The angle at $\tilde{z}$ in the triangle $0 \tilde{z} \tilde{y}$ in $\mathbb{R}^{2}$ is therefore acute, and so (9.1) follows (even if the triangle degenerates):

$$
d_{N}\left(p_{\theta}(y), p_{\theta}\left(y^{\prime}\right)\right) \leq\left|\left(p_{\theta}(y)\right)^{\sim}-\left(p_{\theta}\left(y^{\prime}\right)\right)^{\sim}\right| \leq|\tilde{y}-\tilde{z}| \leq\left|\tilde{y}-\tilde{y}^{\prime}\right|=d_{N}\left(y, y^{\prime}\right) .
$$

The contractivity of $p_{\theta}$ implies that

$$
E\left(p_{\theta} \circ \phi\right) \leq E(\phi) .
$$

In terms of Definition 1 this follows from [EF, Corollary 9.1]. Using instead Definition 2 we employ a quasicontinuous version of $\phi$; then $|\phi|\left(=d_{N}(\phi(\cdot), q)\right), \theta \circ|\phi|$, and $p_{\theta} \circ \phi=(\theta \circ|\phi|) \phi$ are likewise quasicontinuous; furthermore, each $\phi^{\alpha} \in W^{1,2}(X)$. The components $p_{\theta}^{\alpha} \circ \phi=(\theta \circ|\phi|) \phi^{\alpha}$ of $p_{\theta} \circ \phi$ are bounded in absolute value by $(\theta \circ|\phi|)|\phi| \leq r$. They are of class $W^{1,2}(X)$ by Definition 2 and the chain rule [EF] Lemma 5.2]) because $(\theta \circ|y|) y^{\alpha} \in C^{1}\left(\mathbb{R}^{n}\right)$ has bounded gradient (and takes the value 0 at 0$)$.

Since $p_{\theta}$ is contractive, the $n \times n$ matrix $A$ with entries

$$
a_{\alpha \beta}=h_{\alpha \beta}-\left(h_{i j} \circ p_{\theta}\right)\left(\partial_{\alpha} p_{\theta}^{i}\right)\left(\partial_{\beta} p_{\theta}^{j}\right)
$$

(as functions of $y \in N$ ) is positive semidefinite and hence of the form $A=C^{*} C$, i.e., $a_{\alpha \beta}=\sum_{k=1}^{n} c_{k \alpha} c_{k \beta}$. It follows that

$$
e(\phi)-e\left(p_{\theta} \circ \phi\right)=\left(a_{\alpha \beta} \circ \phi\right)\left\langle\nabla \phi^{\alpha}, \nabla \phi^{\beta}\right\rangle=\sum_{k=1}^{n}\left|\left(c_{k \alpha} \circ \phi\right) \nabla \phi^{\alpha}\right|^{2} \geq 0
$$

$\mu$-a.e. in $X$, and (9.3) ensues by integration.

Allowing $r>0$ to vary, write $p_{\theta_{r}} \circ \phi=\phi_{r}$. Since $t \theta_{r}(t) \leq r$, we have $\phi_{r}(X) \subset$ $B_{N}(q, r)$. Consequently, by Part (a) of the proposition, $E\left(\phi_{r}\right)$ in the sense of Definition 1 equals $c_{m}$ times $E\left(\phi_{r}\right)$ in the sense of Definition 2. The corresponding relation between the two energies $E(\phi)$ follows from [EF, Theorem 9.2 (a)] in case $E(\phi)=\infty$ in the sense of Definition 2. In the remaining case where $E(\phi)<\infty$ (Definition 2) we show that

$$
E(\phi)=\lim _{r \rightarrow \infty} E\left(\phi_{r}\right)
$$

in either sense, whence the asserted relation between the two energies $E(\phi)$. And that will further imply the same relation $\mu$-a.e. between the energy densities $e(\phi)$ for the two definitions, again according to [EF, Theorem 9.2 (a)], and thus complete the proof of Proposition 2.

To prove the remaining equation (9.4), first in the setting of Definition 2, note that each $\phi^{\alpha} \in W^{1,2}(X) \subset L^{2}(X)$, and hence $d_{N}(\phi, q)=|\phi| \in L^{2}(X)$; that is, $\phi \in L^{2}(X, N)$. Because $t \theta_{r}(t) \nearrow t$ as $r \nearrow \infty$ it follows that

$$
\int_{X} d_{N}^{2}\left(\phi, \phi_{r}\right) d \mu=\int_{X}\left(|\phi|-|\phi| \theta_{r}(|\phi|)\right)^{2} d \mu \searrow 0,
$$


and so $\phi_{r} \rightarrow \phi$ in $L^{2}(X, N)$. Invoking (9.3) and [EF, Lemma 9.1, Remark 9.6] we thus arrive at (9.4) for either definition:

$$
E(\phi) \leq \liminf _{r \rightarrow \infty} E\left(\phi_{r}\right) \leq \limsup _{r \rightarrow \infty} E\left(\phi_{r}\right) \leq E(\phi) .
$$

\section{Proof of Lemma 3}

We may assume that $X$ is a standard $m$-simplex in $\mathbb{R}^{m}$. Suppose first that $g$ is smooth. Let $\phi_{0}, \phi_{1}: X \rightarrow N$ have finite energy in the sense of Definition 2, and hence also in the sense of Definition 1, by Proposition 2 (b) proved above. It then follows from [EF, eq. (11.1)] by application of [EF, Corollary 9.2] that

$$
e\left(\phi_{t}\right) \leq(1-t) e\left(\phi_{0}\right)+t e\left(\phi_{1}\right)-t(1-t)\left|\nabla d_{N}\left(\phi_{0}, \phi_{1}\right)\right|^{2}
$$

$\mu$-a.e. in $X$, where $e(\cdot)$ denotes energy density in the sense of Definition 2, equal to $1 / c_{m}$ times the energy density from Definition 1 . We shall extend (10.1) by approximation to the case where $g$ is merely bounded measurable, and as always with elliptic bounds $\Lambda_{X}^{-2}, \Lambda_{X}^{2}$; cf. [EF, p. 48]. We may of course assume that $g$ is defined and measurable on all of $X$ (e.g. $g(x)=$ the $m \times m$ unit matrix for $\left.x \in \mathbb{R}^{m} \backslash X\right)$.

Consider the set $G$ of all symmetric $m \times m$ matrices $\left(g_{i j}\right)$ such that (with summation over $i, j \in\{1, \ldots, m\})$

$$
\Lambda_{X}^{-2} \leq g_{i j} \xi^{i} \xi^{j} \leq \Lambda_{X}^{2}
$$

for all unit vectors $\xi=\left(\xi^{1}, \ldots, \xi^{m}\right)$ in $\mathbb{R}^{m}$. Because $G \subset \mathbb{R}^{M}, M=m(m+1) / 2$, the given measurable Riemannian metric $g: \mathbb{R}^{m} \rightarrow G$ may be viewed as a map of class $L^{1}\left(\mathbb{R}^{m}, \mathbb{R}^{M}\right)$. By convolution with a smooth approximate identity $\kappa$ of $L^{1}\left(\mathbb{R}^{m}\right), g \in L^{1}\left(\mathbb{R}^{m}, G\right)$ is approximated in $L^{1}\left(\mathbb{R}^{m}, \mathbb{R}^{M}\right)$ by smooth maps $\kappa * g$ of class $L^{1}\left(\mathbb{R}^{m}, G\right)$ because $G$ is convex and compact in $\mathbb{R}^{M}$. There is therefore a sequence of smooth maps $g_{p}=\kappa_{p} * g: \mathbb{R}^{m} \rightarrow G$ converging pointwise to $g \mu$-a.e. in $\mathbb{R}^{m}$. Hence (10.1) follows from the same inequality with $g$ replaced by $g_{p}$ (now considered on $X$ only), by letting $p \rightarrow \infty$, noting that, for each chart $V \rightarrow \mathbb{R}^{n}$,

$$
\begin{aligned}
& e_{p}\left(\phi_{t}\right)=g_{p}^{i j}\left(h_{\alpha \beta} \circ \phi_{t}\right) \partial_{i} \phi_{t}^{\alpha} \partial_{j} \phi_{t}^{\beta} \quad \mu \text {-a.e. in }\left(\phi_{t}\right)^{-1}(V), t \in[0,1], \\
& \left|\nabla_{p} d_{N}\left(\phi_{0}, \phi_{1}\right)\right|^{2}=g_{p}^{i j} \partial_{i}\left[d_{N}\left(\phi_{0}, \phi_{1}\right)\right] \partial_{j}\left[d_{N}\left(\phi_{0}, \phi_{1}\right)\right] \quad \mu \text {-a.e. in } X,
\end{aligned}
$$

the energy density $e_{p}$ and the gradient $\nabla_{p}$ referring to the Riemannian metric $g_{p}$ on $X$. The version of (10.1) for the total energies now follows by integration.

\section{REFERENCES}

[A1] Alexandrov, A.D., A theorem on triangles in a metric space and some of its applications, (In Russian), Trudy Math. Inst. Steklov 38 (1951), 5-23. MR 14:198a

[A2] Alexandrov, A.D., Über eine Verallgemeinerung der Riemannschen Geometrie, Schr. Forschungsinst. Math. Berlin 1 (1957), 33-84. MR 19:304h

[AB1] Alexander, S.B., and R.L. Bishop, The Hadamard-Cartan theorem in locally convex metric spaces, L'Enseignement Math. 36 (1990), 309-320. MR 92c:53044

[AB2] Alexander, S.B., and R.L. Bishop, Comparison theorems for curves of bounded geodesic curvature in metric spaces of curvature bounded above, Diff. Geom. Appl. 6 (1996), 67-86. MR 97a:53051

[De] Deny, J., Méthodes hilbertiennes en théorie du potentiel, Potential Theory, C.I.M.E, Stresa 1969, Ed. Cremonese, Roma, 1970, pp. 121-201. MR 44:1833

[EF] Eells, J., and B. Fuglede, Harmonic maps between Riemannian polyhedra, Cambridge Tracts in Mathematics No. 142, Cambridge University Press, 2001. MR 2002h:58017 
[EP] Eells, J., and J.C. Polking, Removable singularities of harmonic maps, Indiana Univ. Math. J. 33 (1984), 859-871. MR 86e:58018

[F1] Fuglede, B., Finely Harmonic Functions, Lecture Notes in Math., No. 289, Springer, Berlin, 1972. MR 56:8883

[F2] Fuglede, B., Fonctions BLD et fonctions finement surharmoniques, Sém. Théorie du Potentiel Paris, No. 6, Lecture Notes in Math. No. 906, Springer, Berlin, 1982, pp. 126157. MR 84b:31009

[F3] Fuglede, B., Hölder continuity of harmonic maps from Riemannian polyhedra to spaces of upper bounded curvature, Calc. Var. 16 (2003), 375-403.

[F4] Fuglede, B., Finite energy maps from Riemannian polyhedra to metric spaces, Ann. Acad. Sci. Fenn. Math. 28 (2003), no. 2, 433-458.

[F5] Fuglede, B., Dirichlet problems for harmonic maps from regular domains, Manuscript.

[GH] Giaquinta, M. and S. Hildebrandt, A priori estimates for harmonic mappings, J. Reine Angew. Math. 336 (1982), 124-164. MR 84b:58035

[Her] Hervé, R.-M., Un principe du maximum pour les sous-solutions locales d'une équation uniformément elliptique de la forme $L u=-\sum_{i} \partial / \partial x_{i}\left(\sum_{j} a_{i j} \partial u / \partial x_{j}\right)=0$, Ann. Inst. Fourier (Grenoble) 14 (2) (1964), 493-508. MR 30:5040

[HKW] Hildebrandt, S., H. Kaul, and K.O. Widman, An existence theorem for harmonic mappings of Riemannian manifolds, Acta Math. 138 (1977), 1-16. MR 55:6478

[Ish] Ishihara, T., A mapping of Riemannian manifolds which preserves harmonic functions, J. Math. Kyoto Univ. 19 (1979), 215-229. MR 80k:58045

[JK] Jäger, W., and H. Kaul, Uniqueness and stability of harmonic maps and their Jacobi fields, Manuscripta Math. 28 (1979), 269-291. MR 80j:58030

[Jo1] Jost, J., Equilibrium maps between metric spaces, Calc. Var. 2 (1994), 173-204. MR 98a:58049

[Jo2] Jost, J., Generalized Dirichlet forms and harmonic maps, Calc. Var. P.D.E. 5 (1997), 1-19. MR 98f:31014

[KM] Kilpeläinen, T., and J. Malý, Supersolutions to degenerate elliptic equations on quasi open sets, Comm. P.D.E. 17 (1992), 371-405. MR 93g:31022

[KS] Korevaar, N.J., and R.M. Schoen, Sobolev spaces and harmonic maps for metric space targets, Comm. Anal. Geom. 1 (1993), 561-659. Reprinted as Chapter X in Lectures on harmonic maps, by R. Schoen and S.T. Yau, Conf. Proc. and Lecture Notes in Geometry and Topology, Vol. II (1997), 204-310. MR 95b:58043

[La] Landkof, N.S., Foundations of Modern Potential Theory, Springer, Berlin, 1972. MR 50:2520

[R] Reshetnyak, Y.G., Inextensible [=Non-expansive] mappings in a space of curvature no greater than K, Siberian Math. J. 9 (1968), 683-689. MR 39:6235

[Sbg] Schoenberg, I.J., Remarks to Maurice Fréchet's article: Sur la définition axiomatique d'une classe d'espaces distancés vectoriellement applicables sur l'espace de Hilbert, Ann. Math. 36 (1935), 724-732.

[Se1] Serbinowski, T., Boundary regularity of harmonic maps to nonpositively curved metric spaces, Comm. Anal. Geom. 2 (1994), 1-15. MR 95k:58050

[Se2] Serbinowski, T., Harmonic maps into metric spaces with curvature bounded above, Thesis, Univ. Utah, 1995.

Department of Mathematics, Universitetsparken 5, DK-2100 Copenhagen $\varnothing$, Denmark

E-mail address: fuglede@math.ku.dk 